\title{
Mixed Transition Systems Revisited
}

\author{
Ou Wei*, Arie Gurfinkel ${ }^{\dagger}$, and Marsha Chechik* \\ *University of Toronto. \{owei, chechik\}@cs.toronto.edu \\ ${ }^{\dagger}$ Software Engineering Institute, Carnegie Mellon University. arie@sei.cmu .edu
}

\begin{abstract}
A variety of partial modeling formalisms, aimed to capture and reason about abstractions, have been proposed. Some, e.g., Kripke Modal Transition Systems (KMTSs) put strong restrictions on necessary and possible behaviours. Some, e.g., Mixed Transition Systems (MixTSs), relax these restrictions. Yet others, e.g., Generalized Kripke MTSs (GKMTSs), allow hypertransitions.

In this paper, we aim to understand trade-offs between these formalisms w.r.t. their applicability to symbolic model-checking. We establish that these formalisms have the same expressive power while differing in succinctness. We also measure the analyzability of these formalisms, measured as the precision of computing compositional semantics of temporal logic formulas. We show that the standard compositional semantics is not preserved between equivalent GKMTSs and MixTSs, and introduce a novel semantics, called reduced, which remains compositional while being both more precise than the standard one and preserved by the semantic equivalence.

We also present a symbolic algorithm to compute the reduced semantics for MixTS models built via predicate abstractions and report on our experience using it in practice.
\end{abstract}

\section{INTRODUCTION}

Abstraction is the key to scaling model-checking to industrial-sized problems. Typically, a large (or infinite) concrete system is approximated by a smaller abstract system via abstracting the concrete states, analyzing the resulting abstract system, and lifting the result back to the concrete system. The interpretation of the result depends on the type of property being checked, and the type of the abstraction used. The two common abstraction schemes are over-approximation - the abstract system contains more behaviours than the concrete one and thus preserves universal properties, and under-approximation - the abstract system contains less behaviours than the concrete one and thus preserves existential properties. Preserving arbitrary properties (e.g., full $\mu$-calculus, $L_{\mu}$, [15]) requires combining over- and under-approximation into a single model $[5,16]$. This is done via using two types of transitions, may and must, representing possible (or over-approximating), and necessary (or under-approximating) behaviours, respectively. We refer to such models as partial. Temporal properties over partial models are interpreted using the 3-valued semantics: a property can be either true, false, or unknown.

A variety of partial modeling formalisms have been developed, forming three separate families. The first is Kripke Modal Transition Systems (KMTSs) [14] and their equivalent variants, Modal TSs [16], Partial Kripke Structures (PKSs) [2], and 3-valued KSs [3]. It requires that every must transition is also a may transition. KMTSs were first introduced as computational models for partial specifications of reactive systems [16], and later adapted for model-checking [2,3, 14]. The second family is Mixed Transition Systems (MixTSs) [5], or, equivalently, Belnap TSs [12]. It places no restrictions on the relationship between may and must transitions and thus extends KMTSs. MixTSs where introduced in [5] as abstract models for $L_{\mu}$, and have been combined with predicate abstraction and software model-checking in [11]. The third family is Generalized KMTSs (GKMTSs) [20], or, equivalently, Abstract TSs [7] and Disjunctive MTSs [17]. It extends MixTSs by allowing must hyper-transitions, i.e., transitions into sets of states. Both MixTSs and KMTSs have been used in practical symbolic model-checkers (e.g., [3,11,13]), while the direct use of GKMTSs has been hampered by the difficulty of compactly encoding hyper-transitions into BDDs.

In this paper, we compare the three families w.r.t. their suitability as the "right" formalism for symbolic model-checking of partial models. Our basis of comparison is (i) the expressive power of the formalisms (i.e., what can be modeled, what abstraction can be captured) (ii) analyzability of the formalisms (i.e., the cost and precision of evaluating temporal logic).

We show that MixTSs, KMTSs and GKMTSs are equally expressive: for any partial model $M$ expressed in one formalism, there exists a partial model $M^{\prime}$ in the other s.t. $M$ and $M^{\prime}$ approximate the same set of concrete systems. Thus, neither hyper-transitions nor restrictions on may and must transitions affect expressiveness. They do, however, affect the size of the models: a GKMTS can be modeled by a MixTS of smaller or equal size (the reduction can be exponential), and MixTSs are more succinct than KMTSs. Dams and Namjoshi have showed that all of the above partial models are subsumed by tree automata [6]. Our work completes the picture by showing the expressive equivalence between those formalisms.

A semantics of temporal logic is called compositional if it is defined inductively on the syntax of the logic. We refer to the typical compositional semantics of $L_{\mu}$ on partial models as standard (SCS). We show that GKMTSs are more precise than MixTSs (and, hence, KMTSs), w.r.t. SCS. That is, a GKMTS can prove/disprove more properties under SCS than a MixTS obtained by a semantics-preserving translation. This is significant since in practice partial models are evaluated w.r.t. compositional semantics. We propose a novel alternative semantics, called reduced (RCS), which remains compositional (and tractable) and is more precise than SCS. We show that GKMTSs and MixTSs are equivalent w.r.t. RCS. Thus, we argue that MixTSs offer a more compact and more versatile alternative to GKMTSs, supporting efficient symbolic 
compositional model checking.

To show the practical impact of the above result, we present a symbolic algorithm to compute the reduced semantics of MixTS models constructed using predicate abstraction. We describe our implementation and evaluate it empirically against the standard compositional semantics.

The rest of the paper is organized as follows. Sec. II reviews the necessary background on partial models and abstraction. In Sec. IV, we show that KMTSs, MixTSs and GKMTSs are equally expressive by developing semantics-preserving translations from GKMTSs to MixTSs, and from MixTSs to KMTSs. In Sec. V, we introduce a new reduced compositional semantics (RCS) for $L_{\mu}$. In Sec. VI, we present a symbolic algorithm to compute RCS in the context of predicate abstraction, and report on our experience with this algorithm in Sec. VII. We conclude the paper in Sec. VIII with the summary of the paper, and comparison with related work.

\section{PRELIMINARIES}

In this section, we review several complete and partial modeling formalisms, and their use for abstraction.

\section{A. Complete and Partial Models}

A statespace of a partial transition system is a tuple $\left\langle S, \preceq_{S}\right\rangle$, where $S$ is a set of states, and $\preceq_{S}$ is a partial order on $S$. Intuitively, $s_{1} \preceq_{S} s_{2}$ means that $s_{1}$ is less informative (more partial) than $s_{2}$.

Def. 1 (Partial TSs) $[1,5,14,20] A$ Generalized Kripke Modal Transition System (GKMTS) is a tuple $M=\left\langle\left\langle S, \preceq_{S}\right\rangle\right.$, $\left.R^{\text {may }}, R^{\text {must }}\right\rangle$, where $\left\langle S, \preceq_{S}\right\rangle$ is the statespace, and $R^{\text {may }} \subseteq S \times$ $S, R^{\text {must }} \subseteq S \times 2^{S}$ are the may and must transition relations, respectively. A Mixed TS (MixTS) is a GKMTS s.t. $R^{\text {must }} \subseteq$ $S \times S . A$ Kripke Modal TS (KMTS) is a MixTS s.t. $R^{\text {must }} \subseteq$ $R^{\text {may }}$. A Boolean TS (BTS) is a KMTS s.t. $R^{\text {may }}=R^{\text {must }}$.

We write $s \stackrel{\text { may }}{\longrightarrow} t$ for $(s, t) \in R^{\text {may }}, s \stackrel{\text { must }}{\longrightarrow} t$, and $s \stackrel{\text { must }}{\longrightarrow} Q$ for $(s, t) \in R^{\text {must }}$ and $(s, Q) \in R^{\text {must }}$, respectively. Intuitively, may and must transitions represent possible and necessary behaviours, respectively. For example, a BTS is complete (i.e., not partial) since every may behaviour is also a must behaviour.

Let $A P$ be a set of atomic propositions, $\operatorname{Lit}(A P)$ be a set of literals of $A P$, and $S$ be a statespace. A state labeling is a function $L: S \rightarrow 2^{A P}$ that assigns to each state $s$ a set of literals that are true in $s$. A TS $M$ together with a labeling $L$, written $\langle M, L\rangle$, is called a model. $L$ is defined over literals. Thus, if $p \in L(s)$, we say that $p$ is true in $s$; if $\neg p \in L(s)$ $-p$ is false in $s$; otherwise, the value of $p$ is unknown. We require that a state labeling is locally consistent, i.e., at most one of $p$ and $\neg p$ belongs to $L(s)$; and monotone w.r.t. $\preceq_{S}$, i.e., $s_{1} \preceq_{S} s_{2} \Rightarrow L\left(s_{1}\right) \subseteq L\left(s_{2}\right)$.

In this paper, we use the modal $\mu$-calculus [15] $\left(L_{\mu}\right)$ as our temporal logic. It is defined as the set of all formulas satisfying the following grammar:

$$
\varphi::=p|\neg \varphi| \varphi \wedge \varphi|\nabla \varphi| \mu Z \cdot \varphi(Z),
$$

where $p$ is an atomic proposition, and $Z$ a fixpoint variable. Furthermore, $Z$ in $\mu Z \cdot \varphi(Z)$ must occur under the scope of an even number of negations. Additional operations are defined as abbreviations: $\varphi \vee \psi \triangleq \neg(\neg \varphi \wedge \neg \psi), \square \varphi \triangleq \neg \diamond \neg \varphi$, $\nu Z \cdot \varphi(Z) \triangleq \neg \mu Z \cdot \neg \varphi(\neg Z)$.

Let $\mathcal{M}=\langle M, L\rangle$ be a model, where $M=\left\langle S, R^{\text {may }}, R^{\text {must }}\right\rangle$, and $\varphi$ be an $L_{\mu}$ formula. An interpretation (or semantics) of $\varphi$ over $\mathcal{M}$, denoted $\|\varphi\|^{\mathcal{M}}$, is given by a pair $\langle U, O\rangle$, where $U, O \subseteq S$. Intuitively, $U$ is the set of states that satisfy $\varphi$, and $O$ is the set of states that are "not known to refute" $\varphi$. Thus, $\varphi$ is true in $U$, false in $S \backslash O$ and unknown in $O \backslash U$. We call $U$ and $O$ the under- and the over-approximation of $\varphi$, respectively.

The semantics of $L_{\mu}$ is called compositional if it is inductive on the syntax of the logic. We refer to the commonly used compositional semantics as standard (SCS). In the definition, we use the following notation. Let $e=\langle U, O\rangle$. We write $\mathrm{U}(e)$ and $\mathrm{O}(e)$ to denote $U$ and $O$, respectively. We use operators $\sqcap$ and $\sim$ defined as follows: $\sim\langle U, O\rangle \triangleq\langle\bar{O}, \bar{U}\rangle$, and $\left\langle U_{1}, O_{1}\right\rangle \sqcap$ $\left\langle U_{2}, O_{2}\right\rangle \triangleq\left\langle U_{1} \cap U_{2}, O_{1} \cap O_{2}\right\rangle$.

Def. 2 (SCS) $[1,5,12,14,20]$. Let $\mathcal{M}=\left\langle M, L_{M}\right\rangle$ be a model, $M=\left\langle S, R^{\text {may }}, R^{\text {must }}\right\rangle$, Var a set of fixpoint variables, and $\sigma: \operatorname{Var} \rightarrow 2^{S} \times 2^{S}$. The standard compositional semantics (SCS) of $\varphi \in L_{\mu}$ is:

$$
\begin{aligned}
\|p\|_{c, \sigma}^{\mathcal{M}} & \triangleq\left\langle\left\{s \mid p \in L_{M}(s)\right\},\left\{s \mid \neg p \notin L_{M}(s)\right\}\right\rangle \\
\|\neg \varphi\|_{c, \sigma}^{\mathcal{M}} & \triangleq \sim\|\varphi\|_{c, \sigma}^{\mathcal{M}} \quad\|Z\|_{c, \sigma}^{\mathcal{M}} \triangleq \sigma(Z) \\
\|\varphi \wedge \psi\|_{c, \sigma}^{\mathcal{M}} & \triangleq\|\varphi\|_{c, \sigma}^{\mathcal{M}} \sqcap\|\psi\|_{c, \sigma}^{\mathcal{M}} \\
\|\nabla \varphi\|_{c, \sigma}^{\mathcal{M}} \triangleq & \left\langle p r e_{\mathrm{U}}\left(\mathrm{U}\left(\|\varphi\|_{c, \sigma}^{\mathcal{M}}\right)\right), p r e_{\mathrm{O}}\left(\mathrm{O}\left(\|\varphi\|_{c, \sigma}^{\mathcal{M}}\right)\right)\right\rangle \\
\|\mu Z \cdot \varphi\|_{c, \sigma}^{\mathcal{M}} \triangleq & \left\langle l f p^{\sqsubseteq}\left(\lambda Q \cdot \mathrm{U}\left(\|\varphi\|_{c, \sigma[Z \mapsto Q]}^{\mathcal{M}}\right)\right),\right. \\
& \left.\quad l f p^{\sqsubseteq}\left(\lambda Q \cdot \mathrm{O}\left(\|\varphi\|_{c, \sigma[Z \mapsto Q]}^{\mathcal{M}}\right)\right)\right\rangle
\end{aligned}
$$

where $Z \in V$ ar, lfp is the least fixpoint, and the pre-image operators pre $\mathrm{U}$ and pre $\mathrm{O}$ are defined as follows:

$$
\begin{aligned}
& \operatorname{pre}_{\mathrm{U}}(Q) \triangleq \begin{cases}\{s \mid \exists t \in Q \cdot s \stackrel{\text { must }}{\longrightarrow} t\} & \text { if } M \text { is a MixTS } \\
\{s \mid \exists U \subseteq Q \cdot s \stackrel{\text { must }}{\longrightarrow} U\} & \text { if } M \text { is a GKMTS }\end{cases} \\
& \operatorname{pre}_{\mathrm{O}}(Q) \triangleq\{s \mid \exists t \in Q \cdot s \stackrel{\text { may }}{\longrightarrow} t\}
\end{aligned}
$$

\section{B. Partial Models and Abstraction}

A concrete statespace $C$ is a set of states. An abstract statespace approximating $C$ is a set of states $S$ together with a soundness relation $\rho: C \times S$, where $(c, s) \in \rho$ means that $s \rho$-approximates $c$. $\rho$ induces a concretization function $\gamma(s) \triangleq\{c \mid(c, s) \in \rho\}$, and an approximation ordering $\preceq_{a} \subseteq S \times S$ defined as $s \preceq_{a} t \Leftrightarrow \gamma(s) \supseteq \gamma(t)$. That is, $\gamma(s)$ is the set of all concrete states approximated by $s$, and $s \preceq_{a} t$ if $s$ is less precise (more approximate) than $t$. For a set $Q \subseteq S$, we define $\gamma(Q) \triangleq \cup_{s \in Q} \gamma(s)$. Following [4], we require that $\preceq$ be an partial order, and that $S$ satisfy "the existence of a best approximation":

$\forall c \in C \cdot \exists s \in S \cdot\left(\rho(c, s) \wedge \forall s^{\prime} \in S \cdot \rho\left(c, s^{\prime}\right) \Rightarrow \gamma\left(s^{\prime}\right) \supseteq \gamma(s)\right)$

We use an abstraction function $\alpha: C \rightarrow S$ to map each concrete element to its best approximation. The image of $\alpha$ is denoted by $\alpha[S] \triangleq\{\alpha(c) \mid c \in C\}$.

In our examples, we often use the abstract domain of predicate abstraction. Let $P=\left\{p_{1}, \ldots, p_{n}\right\}$ be a set of $n$ predicates. A conjunction of literals of $P$ is called a monomial; a monomial in which each variable $p_{i}$ appears once (either 
positively or negatively) is called a minterm. We write $\operatorname{Mon}(P)$ and $\operatorname{MT}(P)$ for the set of all monomials and minterms of $P$, respectively. The domain of predicate abstraction is the set $\operatorname{Mon}(P)$. The soundness relation $\rho_{P}$ is defined s.t. $(c, s) \in \rho_{P}$ iff $c$ satisfies all predicates in $s$, i.e., $c \models s$; the abstraction $\alpha_{P}(c) \triangleq\left(\bigwedge_{c \mid=p_{i}} p_{i}\right) \wedge\left(\bigwedge_{c \mid \vDash p_{i}} \neg p_{i}\right) ; \alpha_{P}[\operatorname{Mon}(P)]=\operatorname{MT}(P) ;$ and the approximation ordering is reverse implication.

The approximation relation is extended from a statespace to transition systems using the concept of mixed simulation.

Def. 3 (Mixed Sim.) [5] Let $M_{1}=\left\langle S_{1}, R_{1}^{\text {may }}, R_{1}^{\text {must }}\right\rangle$ and $M_{2}=\left\langle S_{2}, R_{2}^{\text {may }}, R_{2}^{\text {must }}\right\rangle$ be two MixTSs. $H \subseteq S_{1} \times S_{2}$ is a mixed simulation between $M_{1}$ and $M_{2}$ if for any $\left(s_{1}, s_{2}\right) \in H$, the following two conditions hold:

$\exists t_{1} \in S_{1} \cdot s_{1} \stackrel{\text { may }}{\longrightarrow} t_{1} \Rightarrow \exists t_{2} \in S_{2} \cdot s_{2} \stackrel{\text { may }}{\longrightarrow} t_{2} \wedge\left(t_{1}, t_{2}\right) \in H$

$\exists t_{2} \in S_{2} \cdot s_{2} \stackrel{\text { must }}{\longrightarrow} t_{2} \Rightarrow \exists t_{1} \in S_{1} \cdot s_{1} \stackrel{\text { must }}{\longrightarrow} t_{1} \wedge\left(t_{1}, t_{2}\right) \in H$

In this case, we say $M_{2} H$-simulates $M_{1}$, written $M_{2} \preceq_{H} M_{1}$. Intuitively, $M_{2}$ simulates $M_{1}$ whenever $M_{2}$ is less precise about its behaviour than $M_{1}$. This definition generalizes to GKMTSs (c.f., [20]).

Let $C$ and $S$ be a concrete and abstract statespaces, respectively, and $\rho \subseteq C \times S$ be the soundness relation. A partial TS $M$ over $S$ approximates a BTS $B$ over $C$ (or, equivalently $B$ refines $M$ ) iff $M \rho$-simulates $B, M \preceq_{\rho} B$. Let $L_{M}$ and $L_{B}$ be state-labellings for $S$ and $C$, respectively. $L_{M}$ approximates $L_{B}$, denoted $L_{M} \preceq_{\rho} L_{B}$, iff $\rho(c, s) \Rightarrow L_{M}(s) \subseteq L_{B}(c)$. A partial model $\mathcal{M}=\left\langle M, L_{M}\right\rangle$ approximates a concrete model $\mathcal{B}=\left\langle B, L_{B}\right\rangle$ (or, equivalently, $\mathcal{B}$ refines $\mathcal{M}$ ) iff $M \preceq_{\rho} B$, and $L_{M} \preceq_{\rho} L_{B}$.

Theorem 1 [5] Let $\mathcal{B}=\left\langle B, L_{B}\right\rangle$ be a concrete model that refines a partial model $\mathcal{M}=\left\langle M, L_{M}\right\rangle$, and $\varphi \in L_{\mu}$. Then, $\gamma\left(\mathrm{U}\left(\|\varphi\|_{c}^{\mathcal{M}}\right)\right) \subseteq \mathrm{U}\left(\|\varphi\|_{c}^{\mathcal{B}}\right)$, and $\mathrm{O}\left(\|\varphi\|_{c}^{\mathcal{B}}\right) \subseteq \gamma\left(\mathrm{O}\left(\|\varphi\|_{c}^{\mathcal{M}}\right)\right)$.

That is, if $\varphi$ is true (false) at a state $a$ of $\mathcal{M}$, then it is true (false) at all states $\gamma(a)$ of $\mathcal{B}$.

Let $\mathbb{C}[\mathcal{M}]$ be the set of all concrete refinements of $\mathcal{M}$. Intuitively, $\mathbb{C}[\mathcal{M}]$ is the semantic meaning of $\mathcal{M}$. An interpretation of $L_{\mu}$ based on the semantic meaning of a partial model was introduced in [2] as thorough semantics. It is defined as follows: $\|\varphi\|_{t}^{\mathcal{M}}=\langle U, O\rangle$ iff $a \in U \Leftrightarrow \forall \mathcal{B} \in \mathbb{C}[\mathcal{M}] \cdot \gamma(a) \subseteq$ $\mathrm{U}\left(\|\varphi\|_{c}^{\mathcal{B}}\right)$, and $a \notin O \Leftrightarrow \forall \mathcal{B} \in \mathbb{C}[\mathcal{M}] \cdot \gamma(a) \subseteq \mathrm{U}\left(\|\neg \varphi\|_{c}^{\mathcal{B}}\right)$.

To compare different interpretations of $L_{\mu}$, we introduce two ordering relations on $2^{S} \times 2^{S}$. Let $e_{1}=\left\langle U_{1}, O_{1}\right\rangle$ and $e_{2}=\left\langle U_{2}, O_{2}\right\rangle$. We say that $e_{1}$ is less informative than $e_{2}$, written $e_{1} \preceq_{i} e_{2}$ iff $U_{1} \subseteq U_{2}$ and $O_{2} \subseteq O_{1}$. We say that $e_{1}$ is semantically less precise than $e_{2}$, written $e_{1} \preceq_{a} e_{2}$, iff $\gamma\left(U_{1}\right) \subseteq \gamma\left(U_{2}\right)$ and $\gamma\left(\overline{O_{1}}\right) \subseteq \gamma\left(\overline{O_{2}}\right)$.

\section{CONSISTENCY}

\section{A. Two definitions of consistency}

A consistency of a partial model can be defined in two ways: either based on satisfaction of temporal logic formulas (logical consistency), or based on possible concrete refinements (semantic consistency). Here, we formally define the two notions.

A model $\langle M, L\rangle$ is logically consistent if for every $\varphi \in L_{\mu}$, $\mathrm{U}\left(\|\varphi\|_{c}\right) \subseteq \mathrm{O}\left(\|\varphi\|_{c}\right)$. That is, the value of $\varphi$ in a state $s \in S$,
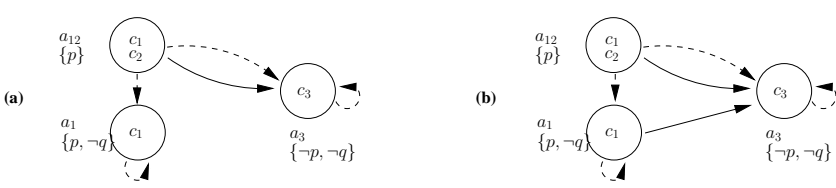

Fig. 1. (a) A semantically inconsistent KMTS $\mathcal{A}_{1}$ where $\gamma\left(a_{1}\right)=\left\{c_{1}\right\}$, $\gamma\left(a_{12}\right)=\left\{c_{1}, c_{2}\right\}$ and $\gamma\left(a_{3}\right)=\left\{c_{3}\right\}$. (b) A monotone KMTS $\mathcal{A}_{2}$ equivalent to $\mathcal{A}_{1}$.

denoted $\|\varphi\|_{c}(s)$, can be defined using 3-valued logic:

$$
\|\varphi\|_{c}(s)= \begin{cases}\mathrm{t} & \text { if } s \in \mathrm{U}\left(\|\varphi\|_{c}\right) \\ \mathrm{f} & \text { if } s \notin \mathrm{O}\left(\|\varphi\|_{c}\right) \\ \mathrm{m} & \text { otherwise }\end{cases}
$$

Def. 4 A transition system $M$ is logically consistent if $\langle M, L\rangle$ is logically consistent for every (consistent) labeling function $L$.

Let $\mathbb{C}[M]$ denote the set of all concrete refinements of $M$. A model $M$ is semantically consistent if $\mathbb{C}[M]$ is not empty.

Def. 5 A transition system $M$ is semantically consistent if $\langle M, L\rangle$ is semantically consistent for every (consistent) labeling function $L$.

There is an obvious relationship between the two notions of consistency.

Theorem 2 If an abstract transition system $M$ is semantically consistent, then it is logically consistent.

The converse of Theorem 2 is not true in general. Filling this gap is the subject of the rest of this section.

\section{B. Checking for consistency}

It is of practical interest of ensure semantic consistency for partial models, e.g., to ensure a specification given by a partial model is implementable in model-based software development. Surprisingly, we found that such conditions have not been precisely defined before. For example, one may think that the condition $R^{\text {must }} \subseteq R^{\text {may }}$ is sufficient to ensure that KMTSs are semantically consistent. However, although this does guarantee that every KMTS is logically consistent, it does not ensure semantic consistency. For example, for the KMTS $\mathcal{A}_{1}$ shown in Figure 1(a), every must transition is also a may transition, but there does not exist a concrete refinement of $\mathcal{A}$ over the states $\left\{c_{1}, c_{2}, c_{3}\right\}$. To see this, suppose such a concrete model $\mathcal{C}$ exists. Because of the transition $a_{12} \stackrel{\text { must }}{\longrightarrow} a_{3}$, there must be a transition $c_{1} \rightarrow c_{3}$ in $\mathcal{C}$. This in turn requires a may transition from $a_{1}$ to $a_{3}$ which does not exists in $\mathcal{A}_{1}$. Similarly, for a GKMTS $\mathcal{A}=\left\langle A, R^{\text {must }}, R^{\text {may }}\right\rangle$, the sufficient condition for logical consistency [7], which requires that every destination of a must hyper-transition intersects with the destination of a may transition from the same state, i.e, $\forall a \in A \cdot \forall U \subseteq A \cdot U \in R^{\text {must }}(a) \Rightarrow U \cap R^{\text {may }}(a) \neq \emptyset$, can be viewed as an analogue of the logical consistency condition for KMTSs, and in general does not ensure semantic consistency either.

The reason that logical and semantic consistency are not equivalent is that they describe consistency for different states- 

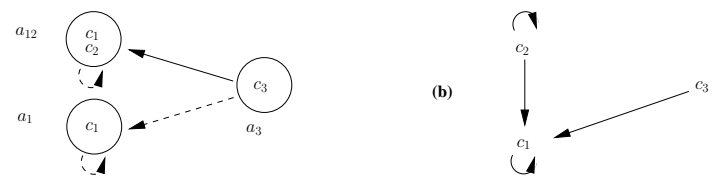

Fig. 2. (a) A consistent MixTS that does not satisfy $R^{\text {must }} \subseteq R^{\text {may }}$. (b) A BTS in that refines a MixTS in (a).

paces - the former ensures consistency of temporal properties for abstract states, and the latter - for concrete states. In general, even if $\mathrm{U}\left(\|\varphi\|_{c}\right) \subseteq \mathrm{O}\left(\|\varphi\|_{c}\right)$ holds for each formula $\varphi$, it may still be possible that there exist concrete states approximated by both of the states in $\mathrm{U}\left(\|\varphi\|_{c}\right)$ and that in $\overline{\mathrm{O}\left(\|\varphi\|_{c}\right)}$, which results in inconsistency on those concrete states. In the previous example, for the formula $\psi \triangleq \diamond \neg p$, we have that $\mathrm{U}\left(\|\psi\|_{c}\right)=\left\{a_{12}\right\} \subseteq \mathrm{O}\left(\|\psi\|_{c}\right)=\left\{a_{12}, a_{3}\right\}$. However, because of $\gamma\left(\mathrm{U}\left(\|\psi\|_{c}\right)\right) \cap \gamma\left(\overline{\left.\mathrm{O}\left(\|\psi\|_{c}\right)\right)}=\left\{c_{1}\right\}\right.$, it implies that $c_{1}$ satisfies both $\psi$ and $\neg \psi$ ! To fix the problem, we add the monotonicity requirement on transition relations (see Def. 9) that guarantees the equivalence between logical and semantic consistency.

Theorem 3 Let $M=\left\langle S_{M}, R_{M}^{\text {must }}, R_{M}^{\text {may }}\right\rangle$ be a monotone (Def. 9) MixTS transition system, then the following are equivalent:

1) $M$ is semantically consistent, i.e., $\mathbb{C}[M]$ is not empty.

2) $M$ is logically consistent, i.e., $\langle M, L\rangle$ is logically consistent for every (consistent) labeling function $L$.

3) for any $a, b_{1} \in S_{M}, a \stackrel{\text { must }}{\longrightarrow} b_{1} \Rightarrow \exists b_{2} \in S_{M} \cdot b_{1} \preceq$ $b_{2} \wedge a \stackrel{\text { may }}{\longrightarrow} b_{2}$.

Intuitively, by adding the monotonicity requirement to a MixTS $\mathcal{A}$, if an abstract state $a_{1}$ is less precise than $a_{2}$, then the truth value of any formula $\varphi$ at $a_{1}$ is less precise than that at $a_{2}$, i.e., $\mathcal{A}, a_{1} \models \varphi \Rightarrow \mathcal{A}, a_{2} \models \varphi$. Therefore, for each concrete state $c$, if the truth value of $\varphi$ is consistent at $\alpha(c)-$ the most precise abstract state approximating $c$, then it is also consistent for $c$.

The next corollary follows immediately from the fact that KMTSs require that every must transition is also a may transition.

Cor. 1 Every monotone KMTS is logically/semantically consistent.

Every MixTS can be translated to an equivalent monotone one without affecting the concrete models it approximates [12]. Thus, Theorem 3 can also be used to check semantic consistency of non-monotone MixTSs. For example, the MixTS $\mathcal{A}_{1}$ in the previous example is equivalent to a monotone MixTS $\mathcal{A}_{2}$ shown in Figure 1(b). It is then easy to check that $\mathcal{A}_{2}$ is not consistent because of the must transition $a_{1} \stackrel{\text { must }}{\longrightarrow} a_{3}$ is not matched by any may transition.

The only difference between KMTSs and MixTSs is that KMTSs require that $R^{\text {must }} \subseteq R^{\text {may }}$. We believe that originally this requirement was introduced to ensure implementability of specifications represented by KMTSs [16]. However, as we have shown, even with this condition a KMTS may still be semantically inconsistent. In the abstraction refinement framework of software model-checking [11], requiring $R^{\text {must }} \subseteq R^{\text {may }}$ makes KMTSs incapable of supporting precise monotone refinement [20] — extra may transitions imposed by this requirement lead to imprecise model checking results. This is not a problem form MixTSs. Note that GKMTS, proposed by Shoham and Grumberg [20] for solving this problem, achieve the same goal by using hyper-transitions. This also ensure that no extra may transitions are added to imprecise states.

\section{EXPRESSIVENESS}

We show that GKMTSs, MixTSs, and KMTSs are expressively equivalent. Two partial TSs $M$ and $M^{\prime}$ are semantically equivalent, denoted $M \equiv_{a} M^{\prime}$, iff they have the same set of concrete refinements. Two modeling formalisms are expressively equivalent iff for every TS $M$ from the one formalism, there exists a TS $M^{\prime}$ from the other, s.t. $M \equiv_{a} M^{\prime}$. The equivalence of the three formalisms is proved by defining semantics-preserving translations between them.

\section{A. GtoM: Translation from GKMTSs to MixTSs}

Here, we present the translation GTOM that converts a GKMTS into a semantically equivalent MixTS. We begin by illustrating the translation on a GKMTS $G_{1}$ in Fig. 3. $G_{1}$ is not a MixTS because of a must hyper-transition $a_{1} \stackrel{\text { must }}{\longrightarrow}\left\{a_{2}, a_{3}\right\}$. This transition ensures that in every concrete BTS refining $G_{1}$, all states in $\gamma\left(a_{1}\right)$, i.e., those satisfying $(x \leq 0 \wedge$ even $(x))$, must have a transition to a state in $\gamma\left(\left\{a_{2}, a_{3}\right\}\right)$, i.e., satisfying $x>0$. No single state of $G_{1}$ represents $x>0$. Thus, this requirement can only be captured either by a hyper transition (as done in $G_{1}$ ), or by extending $G_{1}$ with a new state, say $a_{5}$, such that $\gamma\left(a_{5}\right)=(x>0)$. In the latter case, the must hypertransition $a_{1} \stackrel{\text { must }}{\longrightarrow}\left\{a_{2}, a_{3}\right\}$ can be replaced by a (regular) must transition $a_{1} \stackrel{\text { must }}{\longrightarrow} a_{5}$. The result is a MixTS $M_{1}$ in Fig. 3. Moreover, since $a_{5}$ replaces a "hyper-state" $\left\{a_{2}, a_{3}\right\}$, $a_{5}$ needs to preserve its may behaviours. We do so by adding $a_{5} \stackrel{\text { may }}{\longrightarrow} a_{4}$ and $a_{5} \stackrel{\text { may }}{\longrightarrow} a_{2}$ corresponding to $a_{2} \stackrel{\text { may }}{\longrightarrow} a_{4}$ and $a_{3} \stackrel{\text { may }}{\longrightarrow} a_{2}$, respectively. There are no outgoing must transitions from $a_{5}$ since the existing must transitions from $a_{2}$ and $a_{3}$ are sufficient. $G_{1}$ and $M_{1}$ are semantically equivalent: any BTS that refines $G_{1}$ also refines $M_{1}$, and vice versa.

In our example, a new state was added to encode a hypertransition by a regular one. This isn't always necessary. For example, TSs $G_{2}$ and $M_{2}$ in Fig. 3 are semantically equivalent. The hyper-transition $a_{1} \stackrel{\text { must }}{\longrightarrow}\left\{a_{2}, a_{3}\right\}$ is encoded by $a_{1} \stackrel{\text { must }}{\longrightarrow}$ $a_{3}$ in $M_{2}$ since the hyper-state $\left\{a_{2}, a_{3}\right\}$ is equivalent to an existing state $a_{3}$, i.e., $\gamma\left(\left\{a_{2}, a_{3}\right\}\right)=\gamma\left(a_{3}\right)=(x>0)$.

In summary, a GKMTS $G$ is translated to a MixTS $M$ in two steps: (i) every must hyper-transition $a \stackrel{\text { must }}{\longrightarrow} U$ of $G$ is replaced by a regular must transition $a \stackrel{\text { must }}{\longrightarrow} b$, where $b$ is a (possibly new) state s.t. $\gamma(b)=\gamma(U)$; (ii) may transitions are added for every state introduced in the first step, if any. We formalize this translation below.

Def. 6 (GTOM) Let $G=\left\langle S_{G}, R_{G}^{\text {may }}, R_{G}^{\text {must }}\right\rangle$ be a GKMTS. The translation $\operatorname{GTOM}(G)$ is a MixTS 


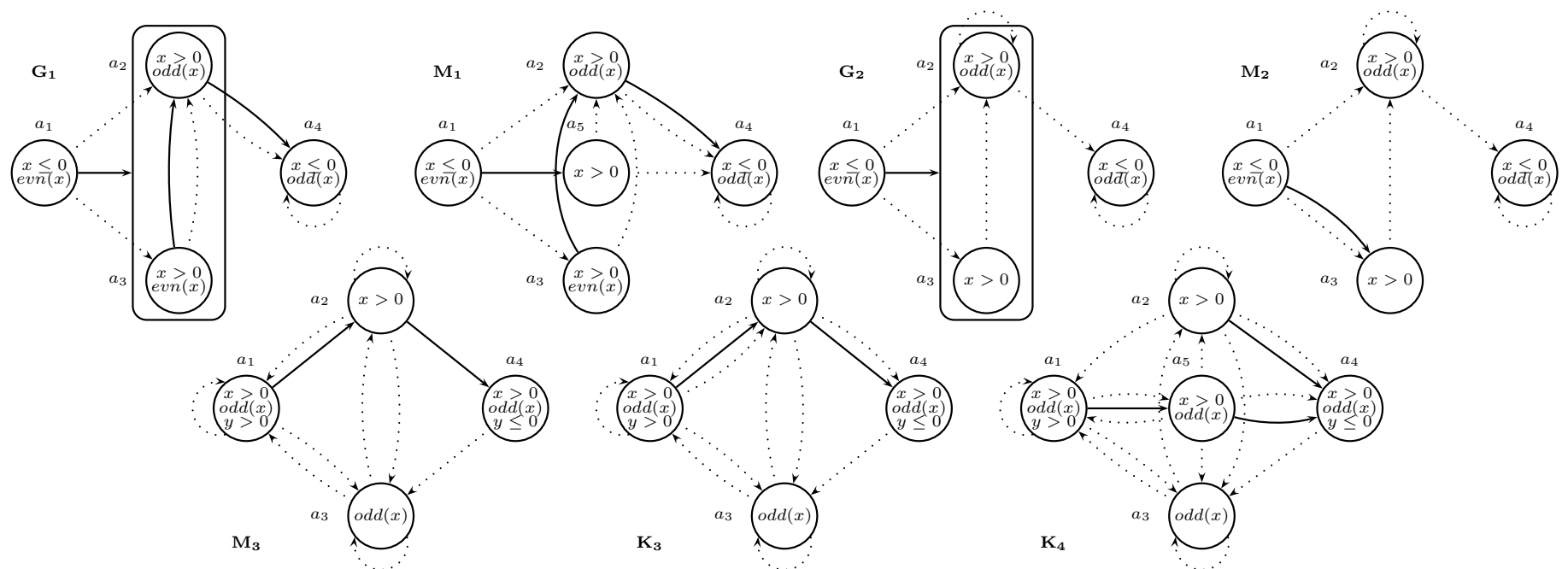

Fig. 3. Two GKMTSs: $G_{1}, G_{2}$; three MixTSs: $M_{1}, M_{2}, M_{3}$; two KMTSs: $K_{3}, K_{4}$. Solid and dashed lines represent must and may transitions, respectively.

$$
\begin{aligned}
& M=\left\langle S_{M}, R_{M}^{\text {must }}, R_{M}^{\text {may }}\right\rangle \text {, such that } \\
& S_{M} \triangleq S_{G} \cup S^{+} \\
& S^{+} \triangleq\left\{a \mid \exists(s, U) \in R_{G}^{\text {must }} \cdot \gamma(a)=\gamma(U) \wedge\right. \\
& \left.\left(\forall t \in S_{G} \cdot \gamma(t) \neq \gamma(U)\right)\right\} \\
& R_{M}^{\text {may }} \triangleq R_{G}^{\text {may }} \cup\left\{(a, b) \mid a \in S^{+} \wedge b \in S_{G} \wedge\right. \\
& \left.\exists s \in S_{G} \cdot(s, b) \in R_{G}^{\text {may }} \wedge \gamma(s) \subseteq \gamma(a)\right\} \\
& R_{M}^{\text {must }} \triangleq\left\{(a, b) \mid a \in S_{G} \wedge b \in S_{M} \wedge\right. \\
& \left.\exists U \subseteq S_{G} \cdot(a, U) \in R_{G}^{\text {must }} \wedge \gamma(b)=\gamma(U)\right\}
\end{aligned}
$$

The translation GTOM is semantics-preserving.

Theorem 4 Let $G$ be a GKMTS, and $M=\operatorname{GTOM}(G)$. Then, $M$ is a MixTS, and $G$ and $M$ are semantically equivalent.

A corollary of Thm. 4 is that GKMTSs and MixTSs are equivalent w.r.t. thorough semantics. Let $L_{G}$ be a labeling function for $G$. We extend the translation GTOM to a GKMTS model $\left\langle G, L_{G}\right\rangle$ such that $\operatorname{GroM}\left(\left\langle G, L_{G}\right\rangle\right) \triangleq\left\langle M, L_{M}\right\rangle$, where $M=\operatorname{GroM}(G)$, and $L_{M}$ is a labeling function for $S_{M}$ defined as follows:

$$
L_{M}(a) \triangleq \begin{cases}L_{G}(a) & \text { if } a \in S_{G} \\ \bigcap_{\left\{s \in S_{G} \mid \gamma(s) \subseteq \gamma(a)\right\}} L_{G}(s) & \text { if } a \in S^{+}\end{cases}
$$

Then $L_{M}$ is well-defined and approximates the same labellings as $L_{G}$. This ensures that $\left\langle G, L_{G}\right\rangle$ and $\left\langle M, L_{M}\right\rangle$ satisfy the same properties under thorough semantics.

Cor. 2 Let $\left\langle G, L_{G}\right\rangle$ be a GKMTS model and $\left\langle M, L_{M}\right\rangle=$ $\operatorname{GroM}\left(\left\langle G, L_{G}\right\rangle\right)$. Then, $\left\langle G, L_{G}\right\rangle$ and $\left\langle M, L_{M}\right\rangle$ are equivalent w.r.t. thorough semantics.

Complexity. We show that the translation GTOM does not increase the size of the model. Let $G$ be a GKTMS with the statespace $S_{G}$, and $M=\operatorname{GroM}(G)$. The size of $G$ is at most $\left|S_{G} \times 2^{S_{G}}\right|$. Each new state added by GTOM corresponds to a subset of $S_{G}$, i.e., $\left|S^{+}\right| \leq\left|2^{S_{G}}\right|$. Furthermore, no transitions between the states in $S^{+}$are added. Thus, the size of $M$ is also at most $\left|S_{G} \times 2^{S_{G}}\right|$.

Sometimes GTOM can reduce a GKMTS exponentially. For example, assume that $S_{G}$ is a disjunctive completion [4], i.e., for every subset $U$ of $S_{G}$ there exists an equivalent element $s$ in $S_{G}$ such that $\gamma(U)=\gamma(s)$. In this case, GToM does not add any new states, i.e., $S^{+}=\emptyset$. This makes the size of the output MixTSs be $\left|S_{G} \times S_{G}\right|$, which is exponentially smaller than that of the input GKMTS.

\section{B. МтоK: Translation from MixTSs to KMTSs}

Below we present the translation MTOK that converts a MixTS into a semantically equivalent KMTS. We begin by illustrating the translation using a MixTS $M_{3}$ in Fig. 3. $M_{3}$ is not a KMTS because of the two must only transitions $a_{1} \stackrel{\text { must }}{\longrightarrow}$ $a_{2}$ and $a_{2} \stackrel{\text { must }}{\longrightarrow} a_{4}$. One way to turn $M_{3}$ into a KMTS is to add may transitions $a_{1} \stackrel{\text { may }}{\longrightarrow} a_{2}$ and $a_{2} \stackrel{\text { may }}{\longrightarrow} a_{4}$, resulting in $K_{3}$ in Fig. 3. However, this transformation is not semanticspreserving, i.e., $K_{3} \not \equiv_{a} M_{3}$. For example, the concrete system ${ }^{1}$

$$
\begin{aligned}
&((y>0) \wedge\left.(x>0) \wedge \operatorname{odd}(x) \wedge x^{\prime}=x+1 \wedge y^{\prime}=y\right) \vee \\
&\left((x>0) \wedge \operatorname{odd}(x) \wedge x^{\prime}=x \wedge y^{\prime}=-1 \times x\right) \vee \\
&\left((x>0) \wedge \neg \operatorname{odd}(x) \wedge x^{\prime}=x+1 \wedge y^{\prime}=-1 \times x\right)
\end{aligned}
$$

refines $K_{3}$, but not $M_{3}$ : the transition $\langle x=1, y=1\rangle \rightarrow\langle x=$ $2, y=1\rangle$ cannot be simulated by any may transition of $M_{3}$.

The must only transition $a_{1} \stackrel{\text { must }}{\longrightarrow} a_{2}$ of $M_{3}$ ensures that in any concrete BTS refining $M_{3}$, all states in $\gamma\left(a_{1}\right)$, i.e., those satisfying $(x>0 \wedge \operatorname{odd}(x) \wedge y>0)$, must have a transition to a state in $\gamma\left(a_{2}\right)$, i.e., satisfying $x>0$. This is further restricted by the may transitions from $a_{1}$ that ensure that states in $\gamma\left(a_{1}\right)$ have transitions only to states in $\gamma\left(\left\{a_{1}, a_{3}\right\}\right)$. Hence, in any BTS refining $M_{3}$, every state in $\gamma\left(a_{1}\right)$ must (and may) have a transition to a state in $\gamma\left(a_{2}\right) \cap \gamma\left(\left\{a_{1}, a_{3}\right\}\right)$.

Intuitively, the must only transition $a_{2} \stackrel{\text { must }}{\longrightarrow} a_{4}$ in $M_{3}$ is equivalent to a pair of may and must transitions from $a_{2}$ to $a_{4}$, since $\gamma\left(a_{4}\right) \cap \gamma\left(\left\{a_{1}, a_{2}, a_{3}\right\}\right)=\gamma\left(a_{4}\right)$. On the other hand, the must only transition $a_{1} \stackrel{\text { must }}{\longrightarrow} a_{2}$ can be equivalently represented by (a) adding a new state $a_{5}$ such that $\gamma\left(a_{5}\right)=\gamma\left(a_{2}\right) \cap$ $\gamma\left(\left\{a_{1}, a_{3}\right\}\right)=(x>0 \wedge$ odd $(x))$, and (b) adding a must and a may transition from $a_{1}$ to $a_{5}$. Moreover, since $a_{5}$ approximates some of the same states as $a_{2}$, i.e., $\gamma\left(a_{5}\right) \subseteq \gamma\left(a_{2}\right), a_{5}$ inherits

\footnotetext{
${ }^{1}$ Unprimed and primed variables represent current- and next-state valuations, respectively.
} 
the transitions from $a_{2}: a_{5} \stackrel{\text { may }}{\longrightarrow} a_{1}, a_{5} \stackrel{\text { may }}{\longrightarrow} a_{2}, a_{5} \stackrel{\text { may }}{\longrightarrow} a_{3}$, $a_{5} \stackrel{\text { must }}{\longrightarrow} a_{4}, a_{5} \stackrel{\text { may }}{\longrightarrow} a_{4}$. The final result is the KMTS $K_{4}$ in Fig. 3, which is semantically equivalent to $M_{3}$.

In summary, a MixTS $M$ is translated to a KMTS $K$ in two steps. First, every must only transition $a \stackrel{\text { must }}{\longrightarrow} b$ of $M$ is replaced by a pair of must and may transitions $a \stackrel{\text { must }}{\longrightarrow} \widehat{a \rightarrow b}$ and $a \stackrel{\text { may }}{\longrightarrow} \widehat{a \rightarrow b}$, where $\widehat{a \rightarrow b}$ is a (possibly new) abstract state such that $\gamma(\widehat{a \rightarrow b})=\gamma(b) \cap \gamma\left(R_{M}^{\text {may }}(a)\right)$. Second, may and must transitions are added for all states introduced in the first step. We formalize this translation below.

Def. 7 (МтоK) Let $M=\left\langle S_{M}, R_{M}^{\text {may }}, R_{M}^{\text {must }}\right\rangle$ be a MixTS. The translation $\operatorname{MTOK}(M)$ is a KMTS $K=\left\langle S_{K}, R_{K}^{\text {may }}, R_{K}^{\text {must }}\right\rangle$, s.t.

$$
\begin{aligned}
& S_{K} \triangleq S_{M} \cup S^{+} \\
& S^{+} \triangleq\left\{\widehat{a \rightarrow b} \mid \exists(a, b) \in\left(R_{M}^{\text {must }} \backslash R_{M}^{\text {may }}\right) \cdot \forall s \in S_{M}\right. \text {. } \\
& \gamma(s) \neq \gamma(\widehat{a \rightarrow b})\} \\
& R_{K}^{\text {may }} \triangleq R_{M}^{\text {may }} \cup \mathrm{REPL} \cup \mathrm{IMAY} \cup \mathrm{IMO} \\
& R_{K}^{\text {must }} \triangleq\left(R_{M}^{\text {must }} \cap R_{M}^{\text {may }}\right) \cup \text { REPL } \cup \text { IMUST } \cup \text { IMO }
\end{aligned}
$$

where

$$
\begin{aligned}
& \mathrm{REPL} \triangleq\left\{(a, \widehat{a \rightarrow b}) \mid \exists(a, b) \in\left(R_{M}^{\text {must }} \backslash R_{M}^{\text {may }}\right)\right\} \\
& \mathrm{IMAY} \triangleq\left\{\left(\widehat{a \rightarrow b}, b^{\prime}\right) \mid \exists a, b, b^{\prime} \in S_{M} .\right. \\
&\left.(a, b) \in\left(R_{M}^{\text {must }} \backslash R_{M}^{\text {may }}\right) \wedge\left(b, b^{\prime}\right) \in R_{M}^{\text {may }} \wedge \widehat{a \rightarrow b} \in S^{+}\right\} \\
& \mathrm{IMUST} \triangleq\left\{\left(\widehat{a \rightarrow b}, b^{\prime}\right) \mid \exists a, b, b^{\prime} \in S_{M} \cdot\right. \\
& \qquad(a, b) \in\left(R_{M}^{\text {must }} \backslash \frac{\left.R_{M}^{\text {may }}\right) \wedge\left(b, b^{\prime}\right) \in\left(R_{M}^{\text {must }} \cap R_{M}^{\text {may }}\right) \wedge}{a \rightarrow b} \in S^{+}\right\}
\end{aligned}
$$

$$
\begin{aligned}
\mathrm{IMO} \triangleq & \left\{\left(\widehat{a \rightarrow b}, \widehat{b \rightarrow b^{\prime}} \mid \exists a, b, b^{\prime} \in S_{M} .\right.\right. \\
& \left.(a, b),\left(b, b^{\prime}\right) \in\left(R_{M}^{\text {must }} \backslash R_{M}^{\text {may }}\right) \wedge \widehat{a \rightarrow b} \in S^{+}\right\}
\end{aligned}
$$

In Def. 7, REPL denotes transitions that replace must only transitions, and IMAY, IMUST and IMO denote transitions from newly added states in $S^{+}$that correspond to may, must, and must only transitions of the original system, respectively. For our example of $\operatorname{MTOK}\left(M_{3}\right)$, we have

$$
\begin{array}{cc}
S^{+}=\left\{a_{5}\right\} & \text { REPL }=\left\{\left(a_{1}, a_{5}\right),\left(a_{2}, a_{4}\right)\right\} \\
\text { IMUST }=\emptyset & \text { IMO }=\left\{\left(a_{5}, a_{4}\right)\right\} \\
\text { IMAY }=\left\{\left(a_{5}, a_{1}\right),\left(a_{5}, a_{2}\right),\left(a_{5}, a_{3}\right)\right\}
\end{array}
$$

The result of the translation MTOK is a KMTS: every must transition is matched by a may transition.

Theorem 5 Let $M$ be a MixTS, and $K=\operatorname{MToK}(M)$. Then $K$ is a KMTS, and $M$ and $K$ are semantically equivalent.

A corollary of Thm. 5 is that MixTSs and KMTSs are equivalent w.r.t. thorough semantics. Let $L_{M}$ be a labeling function for $M$. We extend MTOK to $\left\langle M, L_{M}\right\rangle$ such that $\operatorname{MroK}\left(\left\langle M, L_{M}\right\rangle\right) \triangleq\left\langle K, L_{K}\right\rangle$, where $K=\operatorname{MToK}(M)$, and $L_{K}$ is a labeling function for $S_{K}$ defined as follows:

$$
L_{K}(a) \triangleq \begin{cases}L_{M}(a) & \text { if } a \in S_{M} \\ \bigcup_{\left\{s \in S_{M} \mid \gamma(a) \subseteq \gamma(s)\right\}} L_{M}(s) & \text { if } a \in S^{+}\end{cases}
$$

Then, $L_{K}$ is well-defined and approximates the same labellings as $L_{M}$. This is sufficient to ensure that $\left\langle M, L_{M}\right\rangle$ and $\left\langle K, L_{K}\right\rangle$ satisfy the same properties under thorough semantics.
Cor. 3 Let $\left\langle M, L_{M}\right\rangle$ be a MixTS model and $\left\langle K, L_{K}\right\rangle=$ $\operatorname{MroK}\left(\left\langle M, L_{M}\right\rangle\right)$. Then, $\left\langle M, L_{M}\right\rangle$ and $\left\langle K, L_{K}\right\rangle$ are equivalent w.r.t. thorough semantics.

Complexity. Let $M=\left\langle S_{M}, R_{M}^{\text {may }}, R_{M}^{\text {must }}\right\rangle$ be a MixTS, and $K$ be a KMTS such that $K=\operatorname{MTOK}(M)$. The size of $M$ is bounded by $O\left(\left|S_{M} \times S_{M}\right|\right)$. In the worst case, the translation adds a new state for each must only transition in $R_{M}^{\text {must }} \backslash R_{M}^{\text {may }}$. Therefore, the number of new states $\left|S^{+}\right|$is bounded by $\mid S_{M} \times$ $S_{M} \mid$, and $|K|$ is bounded by $O\left(\left|S_{M} \times S_{M}\right|^{2}\right)$.

MixTSs are more succinct than KMTSs: for a fixed statespace $S$, the set of MixTSs over $S$ is strictly more expressive than the set of KMTSs over $S$. This is true since for every state $t$ added by MTOK, there exists a subset $U \subseteq S$ s.t. $\gamma(t)=\gamma(U)$.

\section{Reduced Compositional Semantics}

GKMTSs and MixTSs are equally expressive: a GKMTS model and its equivalent MixTS model satisfy the same properties under thorough semantics. However, thorough check has exponential complexity. In practice, partial models are evaluated using a more tractable compositional semantics SCS. Unfortunately, GKMTSs are more precise than MixTSs w.r.t. SCS: for any $\varphi \in L_{\mu}$, the value of $\varphi$ in a GKMTS model $\mathcal{G}$ under SCS is more precise than its value in the MixTS model $\mathcal{M}=\operatorname{GroM}(\mathcal{G})$. We propose an alternative semantics, called reduced compositional semantics (RCS). While RCS is defined (and evaluated) inductively on the structure of the formula, it is strictly more precise than SCS. We show that GKMTSs and MixTSs are equivalent w.r.t. RCS.

In Sec. V-A, we illustrate the differences between GKMTSs and MixTSs w.r.t. SCS. We define RCS in Sec. V-B, and show how to compute it effectively in Sec. V-C.

\section{A. Example}

Let $p$ and $q$ denote predicates $x>0$ and $\operatorname{odd}(x)$, respectively. Consider the model $\mathcal{G}_{1}=\left\langle G_{1}, L_{G_{1}}\right\rangle$, where $G_{1}$ is shown in Fig. 3, and $L_{G_{1}}$ is a labeling function that labels each abstract state as shown in Fig. 3. Let $\mathcal{M}_{1}=\left\langle M_{1}, L_{M_{1}}\right\rangle$ be the model obtained from $\mathcal{G}_{1}$ by GTOM, where $M_{1}$ is shown in Fig. 3 and $L_{M_{1}}(s) \triangleq$ if $s=a_{5}$ then $\{p\}$ else $L_{G_{1}}(s)$.

Compare the value of $\varphi \triangleq \diamond(q \vee \neg q)$ under SCS on $\mathcal{G}_{1}$ and $\mathcal{M}_{1}$ :

$$
\begin{aligned}
\|\varphi\|_{c}^{\mathcal{G}_{1}} & =\left\langle\left\{a_{1}, a_{2}, a_{3}\right\},\left\{a_{1}, a_{2}, a_{3}, a_{4}\right\}\right\rangle \\
\|\varphi\|_{c}^{\mathcal{M}_{1}} & =\left\langle\left\{a_{2}, a_{3}\right\},\left\{a_{1}, a_{2}, a_{3}, a_{4}, a_{5}\right\}\right\rangle
\end{aligned}
$$

According to $\mathcal{G}_{1}, \varphi$ is true in all states corresponding to $a_{1}$. According to $\mathcal{M}_{1}$, the value of $\varphi$ is unknown in exactly the same states. Since $\mathcal{M}_{1}=\operatorname{GroM}\left(\mathcal{G}_{1}\right), \mathcal{G}_{1} \equiv_{a} \mathcal{M}_{1}$. Thus, $\mathcal{M}_{1}$ is less precise than $\mathcal{G}_{1}$ under SCS.

Let us examine the above example more carefully. First, there is no precision loss during the evaluation of $q \vee \neg q$ :

$$
\begin{aligned}
& e_{1}=\|q \vee \neg q\|_{c}^{\mathcal{G}_{1}}=\left\langle\left\{a_{1}, a_{2}, a_{3}, a_{4}\right\},\left\{a_{1}, a_{2}, a_{3}, a_{4}\right\}\right\rangle \\
& e_{2}=\|q \vee \neg q\|_{c}^{\mathcal{M}_{1}}=\left\langle\left\{a_{1}, a_{2}, a_{3}, a_{4}\right\},\left\{a_{1}, a_{2}, a_{3}, a_{4}, a_{5}\right\}\right\rangle
\end{aligned}
$$

Since $\gamma\left(\mathrm{U}\left(e_{1}\right)\right)=\gamma\left(\mathrm{U}\left(e_{2}\right)\right)$ and $\gamma\left(\overline{\mathrm{O}\left(e_{1}\right)}\right)=\gamma\left(\overline{\mathrm{O}\left(e_{2}\right)}\right)=$ $\gamma(\emptyset), e_{1} \equiv_{a} e_{2}$. However, there is a subtle difference between them. $q \vee \neg q$ is unknown in state $a_{5}$ of $M_{1}$, even though $\varphi$ 
is true in both $a_{2}$ and $a_{3}$, and $\gamma\left(a_{5}\right)=\gamma\left(a_{2}\right) \cup \gamma\left(a_{3}\right)$. This minor imprecision is then magnified by the $\diamond$ operator.

We note that the precision loss is not limited to tautologies. For example, $\mu Z \cdot(\neg p \wedge q) \vee \diamond Z$, i.e., $E F(\neg p \wedge q)$ in CTL, is true in state $a_{1}$ on $\mathcal{G}_{1}$, but is unknown in the same state of $\mathcal{M}_{1}$.

\section{B. Reduced Compositional Semantics for Partial Models}

In this section, we define the reduced compositional semantics (RCS). The new semantics is compositional and is strictly more precise than SCS. The key idea is to use a reduction operator to eliminate any local imprecision.

Let $S$ be an abstract statespace, and $e, e^{\prime} \in 2^{S} \times 2^{S}$ be two abstract elements. Recall that in the information order $e$ is less than $e^{\prime}$, i.e., $e \preceq_{i} e^{\prime}$, if $\mathrm{U}(e)$ is contained in $\mathrm{U}\left(e^{\prime}\right)$, and $\mathrm{O}(e)$ contains $\mathrm{O}\left(e^{\prime}\right)$. We define the reduction operator as follows: $\operatorname{RED}(e) \triangleq\left\langle\operatorname{RED}_{\mathrm{U}}(U), \operatorname{RED}_{\mathrm{O}}(O)\right\rangle$, where $\operatorname{RED}_{\mathrm{U}}(U) \triangleq$ $\{s \mid \gamma(s) \subseteq \gamma(U)\}$, and $\operatorname{RED}_{\mathrm{O}}(O) \triangleq\{s \mid \gamma(s) \nsubseteq \gamma(\bar{O})\}$. Intuitively, $\operatorname{RED}(e)$ increases $\mathrm{U}(e)$ and decreases $\mathrm{O}(e)$ as much as possible without affecting the semantic meaning of $e$. That is, $\operatorname{RED}(e)$ is the largest element w.r.t. information ordering and semantically equivalent to $e$. For example, consider $\operatorname{RED}\left(e_{2}\right)$, where $e_{2}$ is as defined by $(\star)$. Then,

$$
\begin{aligned}
& e_{3}=\operatorname{RED}\left(e_{2}\right)= \\
&\left\langle\left\{a_{1}, a_{2}, a_{3}, a_{4}, a_{5}\right\},\left\{a_{1}, a_{2}, a_{3}, a_{4}, a_{5}\right\}\right\rangle
\end{aligned}
$$

$e_{3}$ differs from $e_{2}$ only in the addition of $a_{5}$ to $\mathrm{U}\left(e_{3}\right)$. Since $\gamma\left(\mathrm{U}\left(e_{2}\right)\right)=\gamma\left(\mathrm{U}\left(e_{3}\right)\right)$ and $\gamma\left(\overline{\mathrm{O}\left(e_{2}\right)}\right)=\gamma\left(\overline{\mathrm{O}\left(e_{3}\right)}\right) e_{2} \equiv_{a} e_{3}$; but $e_{2}$ is less informative since $\mathrm{U}\left(e_{2}\right) \subset \mathrm{U}\left(e_{3}\right)$.

An element $e=\{U, O\} \in 2^{S} \times 2^{S}$ is monotone iff

$$
s_{1} \preceq_{S} s_{2} \Rightarrow\left(s_{1} \in U \Rightarrow s_{2} \in U \wedge s_{1} \notin O \Rightarrow s_{2} \notin O\right)
$$

$\operatorname{RED}(e)$ is monotone for any $e$, and commutes with propositional operations on monotone elements. That is, let $e$ and $e^{\prime}$ be monotone elements of $2^{S} \times 2^{S}$. Then, $\sim e \equiv_{a} \sim \operatorname{RED}(e)$, and $e \sqcap e^{\prime} \equiv_{a} \operatorname{RED}(e) \sqcap \operatorname{RED}\left(e^{\prime}\right)$.

RCS is defined by applying the RED operator before and after $\diamond$ to prevent it from propagating imprecision.

Def. 8 (RCS) Let $\mathcal{M}=\left\langle M, L_{M}\right\rangle$ be a model, s.t. $M=$ $\left\langle S, R^{\text {may }}, R^{\text {must }}\right\rangle$ and $\sigma: \operatorname{Var} \rightarrow 2^{S} \times 2^{S}$. The reduced compositional semantics of $\varphi \in L_{\mu}$ is defined as follows:

$$
\begin{aligned}
\|p\|_{r, \sigma}^{\mathcal{M}} \triangleq & \left\langle\left\{s \mid p \in L_{M}(s)\right\},\left\{s \mid \neg p \notin L_{M}(s)\right\}\right\rangle \\
\|\neg \varphi\|_{r, \sigma}^{\mathcal{M}} \triangleq & \sim\|\varphi\|_{r, \sigma}^{\mathcal{M}} \quad\|Z\|_{r, \sigma}^{\mathcal{M}} \triangleq \sigma(Z) \\
\|\varphi \wedge \psi\|_{r, \sigma}^{\mathcal{M}} \triangleq & \|\varphi\|_{r, \sigma}^{\mathcal{M}} \sqcap\|\psi\|_{r, \sigma}^{\mathcal{M}} \\
\|\triangleright \varphi\|_{r, \sigma}^{\mathcal{M}} \triangleq & \operatorname{RED}\left(\left\langlep r e_{\mathrm{U}}\left(\operatorname{RED}_{\mathrm{U}}\left(\mathrm{U}\left(\|\varphi\|_{r, \sigma}^{\mathcal{M}}\right)\right)\right),\right.\right. \\
& \left.\left.\operatorname{pre}\left(\operatorname{RED}_{\mathrm{O}}\left(\mathrm{O}\left(\|\varphi\|_{r, \sigma}^{\mathcal{M}}\right)\right)\right)\right\rangle\right) \\
\|\mu Z \cdot \varphi\|_{r, \sigma}^{\mathcal{M}} \triangleq & \left\langle l f p \sqsubseteq\left(\lambda Q \cdot \mathrm{U}\left(\|\varphi\| \|_{r, \sigma[Z \mapsto Q]}^{\mathcal{M}}\right)\right),\right. \\
& \left.l f p^{\sqsubseteq}\left(\lambda Q \cdot \mathrm{O}\left(\|\varphi\|_{r, \sigma[Z \mapsto Q]}^{\mathcal{M}}\right)\right)\right\rangle
\end{aligned}
$$

The only difference between RCS (Def. 8) and SCS (Def. 2) is the semantics of $\diamond$. Since we assume that state-labellings are monotone, applying RED to other operators as well does not improve precision.

Returning to our running example, RCS of $\varphi$ on $\mathcal{M}_{1}$ is computed as follows: RCS of $q, \neg q$, and $q \vee \neg q$ is the same as
SCS. Thus, $\|q \vee \neg q\|_{r}^{\mathcal{M}_{1}}=e_{2}$. To compute $\diamond$, recall from ( $\left.\star \star\right)$ that $\operatorname{RED}\left(e_{2}\right)=e_{3}$; thus,

$$
\|\varphi\|_{r}^{\mathcal{M}_{1}}=\left\langle\left\{a_{1}, a_{2}, a_{3}, a_{5}\right\},\left\{a_{1}, a_{2}, a_{3}, a_{4}, a_{5}\right\}\right\rangle
$$

Hence, $\|\varphi\|_{r}^{\mathcal{M}_{1}}$ is more precise than $\|\varphi\|_{c}^{\mathcal{M}_{1}}$.

Theorem 6 RCS is more precise than SCS: $\|\varphi\|_{c} \preceq_{a}\|\varphi\|_{r}$.

The previous example illustrates another important point: GKMTSs and MixTSs are equivalent w.r.t. RCS. For example, $\|\varphi\|_{r}^{\mathcal{M}_{1}}$ is equivalent to $\|\varphi\|_{r}^{\mathcal{G}_{1}}$. The following theorem formalizes this relationship.

Theorem 7 Let $\mathcal{G}$ be a GKMTS, and $\mathcal{M}=\operatorname{GTOM}(\mathcal{G})$. Then, $\mathcal{G}$ and $\mathcal{M}$ are equivalent w.r.t. $R C S: \forall \varphi \in L_{\mu} \cdot\|\varphi\|_{r}^{\mathcal{G}} \equiv_{a}\|\varphi\|_{r .}^{\mathcal{M}}$

Our new semantics RCS is both compositional and precise enough to make GKMTSs and MixTSs equivalent. However, the RED operator requires comparing concretizations of abstract elements. In practice, this may be impossible or inefficient. We address this limitation next.

\section{Reduced Compositional Semantics for Monotone Models}

We specialize the reduction operator RED of RCS to monotone models.

Def. 9 A MixTS $M=\left\langle S, R^{\text {may }}, R^{\text {must }}\right\rangle$ is monotone iff

$$
\begin{aligned}
\forall s_{1} \preceq_{S} s_{2}, t_{2} \preceq_{S} t_{1} \cdot\left(\left(s_{2}, t_{2}\right) \in R^{\text {may }} \Rightarrow\left(s_{1}, t_{1}\right) \in R^{\text {may }}\right) \wedge \\
\left(\left(s_{1}, t_{1}\right) \in R^{\text {must }} \Rightarrow\left(s_{2}, t_{2}\right) \in R^{\text {must }}\right)
\end{aligned}
$$

A model $\mathcal{M}=\left\langle M, L_{M}\right\rangle$ is monotone iff $M$ is monotone.

Monotone models are as expressive as their regular counterparts [12]. The monotonicity condition simply ensures that all information that can be derived from the existing may and must transitions is made explicit in the model.

For a state $s \in S$, let the upset of $s$ be defined as $\uparrow s \triangleq\left\{t \in \alpha[S] \mid s \preceq_{a} t\right\}$. Then, $\uparrow s$ is the set of all those states in $\alpha[S]$ that are more precise than $s$. For example, let $S_{1}$ be the statespace of $M_{1}$ in Fig. 3. Then, $\alpha\left[S_{1}\right]=\left\{a_{1}, a_{2}, a_{3}, a_{4}\right\}$, and $\uparrow a_{5}=\left\{a_{2}, a_{3}\right\}$. Note that the state $s$ and the set $\uparrow s$ approximate the same set of concrete states, i.e., $\gamma(s)=\gamma(\uparrow s)$. For example, $\gamma\left(\uparrow a_{5}\right)=\gamma\left(a_{5}\right)=(x>0)$.

Let $e=\langle U, O\rangle$ be a monotone element of $2^{S} \times 2^{S}$, and $s \in$ $S$. By monotonicity, $\gamma(s) \subseteq \gamma(U)$ iff $\uparrow s \subseteq U$. Dually, $\gamma(s) \nsubseteq$ $\gamma(\bar{O})$ iff $\uparrow s \nsubseteq \mathbb{O}$. Thus, we define a new operator red as follows: $\operatorname{red}(e) \triangleq\left\langle\operatorname{red}_{\mathrm{U}}(U), \operatorname{red}_{\mathrm{O}}(O)\right\rangle$, where $\operatorname{red}_{\mathrm{U}}(U) \triangleq$ $\{s \mid \uparrow s \subseteq U\}$, and $\left.\operatorname{red}_{\mathrm{O}}(O) \triangleq\{s \mid \uparrow s \nsubseteq \bar{O})\right\}$.

Theorem 8 Let $S$ be an abstract statespace, and e be a monotone element in $2^{S} \times 2^{S}$. Then, $\operatorname{red}(e)=\operatorname{RED}(e)$.

red can be computed effectively since it does not need to concretize abstract elements.

In this section, we have introduced a new compositional semantics RCS, and shown that it is more precise than SCS, and that GKMTSs and MixTSs are equivalent w.r.t. RCS. RCS can be computed effectively on monotone models, which is not restrictive since monotone models are as expressive as their non-monotone counterparts.

\section{Symbolic Computation of RCS USING BDDs}

In this section, we describe a symbolic algorithm RCS that implements the RCS semantics for monotone models 


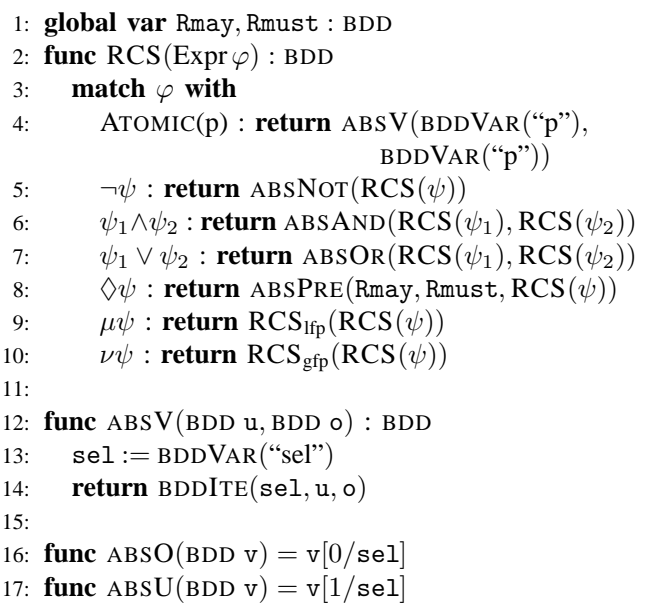

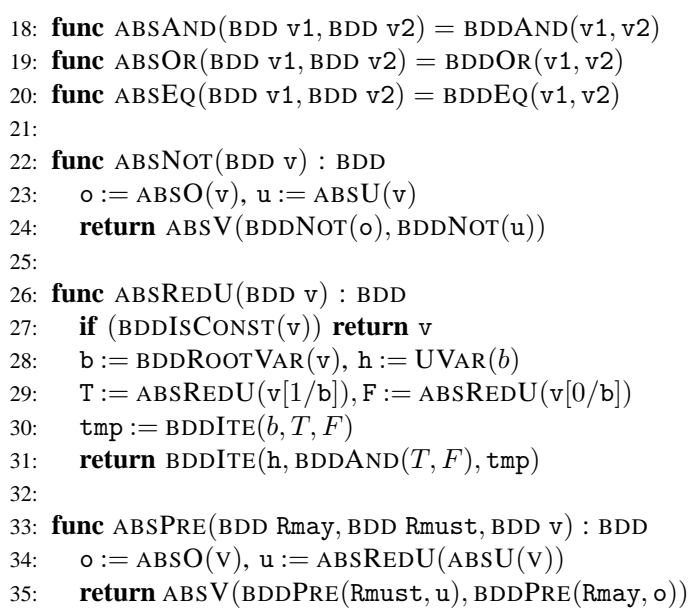

Fig. 4. The RCS algorithm and its supporting functions.

constructed using predicate abstraction. These are the models used by an existing software model-checker [13].

Our implementation is based on the following observation. Let $S$ be an abstract statespace. Then, for any monotone element of $2^{S} \times 2^{S}$ there exists a semantically equivalent element in $2^{\alpha[S]} \times 2^{\alpha[S]}$.

Theorem 9 Let $e_{1}=\left\langle U_{1}, O_{1}\right\rangle$ be a monotone element of $2^{S} \times$ $2^{S}$, and $e_{2}=\left\langle U_{2}, O_{2}\right\rangle$ be in $2^{\alpha[S]} \times 2^{\alpha[S]}$. If $U_{1} \cap \alpha[S]=U_{2}$ and $O_{1} \cap \alpha[S]=O_{2}$, then $e_{1} \equiv{ }_{a} e_{2}$.

This allows us to restrict the algorithm to sets over $\alpha[S]$ instead of sets over $S$. Another consequence of Thm. 9 is that the transition relations can be simplified as well, since we only need the result of the pre-image in the states in $\alpha[S]$.

Theorem 10 Let $R^{\text {may }} \subseteq S \times S$ and $R^{\text {must }} \subseteq S \times S$ be the may and must transition relations of a monotone MixTS, respectively, and $e=\langle U, O\rangle$ be a monotone element of $2^{S} \times 2^{S}$. Define $\hat{U} \triangleq U \cap \alpha[S], \hat{O} \triangleq O \cap \alpha[S], \hat{R}^{\text {must }} \triangleq$ $R^{\text {must }} \cap(\alpha[S] \times S)$, and $\hat{R}^{\text {may }} \triangleq R^{\text {may }} \cap(\alpha[S] \times \alpha[S])$. Then,

$$
\begin{array}{r}
\left\langle\text { pre }\left[R^{\text {must }}\right](\operatorname{RED}(U)), \text { pre }\left[R^{\text {may }}\right]\left(\operatorname{RED}_{\mathrm{O}}(O)\right)\right\rangle \equiv_{a} \\
\left\langle\text { pre }\left[\hat{R}^{\text {must }}\right]\left(\operatorname{RED}_{\mathrm{U}}(\hat{U})\right), \operatorname{pre}\left[\hat{R}^{\text {may }}\right](\hat{O})\right\rangle
\end{array}
$$

The algorithm RCS is shown in Fig. 4. It uses BDDs to symbolically represent and manipulate sets of states and transition relations. Functions that are prefixed with "BDD" are the standard BDD operations. The algorithm works recursively on the structure of the input formula $\varphi$. The fixpoints are computed in the usual way, by iterating until convergence. We describe the details of the implementation below.

Let $P=\left\{p_{1}, \ldots, p_{n}\right\}$ be a set of $n$ predicates. Recall that $\operatorname{Mon}(P)$ denotes the set of monomials over $P$, and $\mathrm{MT}(P)$ - the set of minterms over $P$. Furthermore, $\alpha[\operatorname{Mon}(P)]=\operatorname{MT}(P)$. The input to the algorithm is a MixTS model $\left\langle M, L_{M}\right\rangle$, s.t. $M=\left(S, R^{\text {may }}, R^{\text {must }}\right), S=\operatorname{Mon}(P)$, and $L_{M}(s)=\operatorname{Lit}(s)$, and an $L_{\mu}$ property $\varphi$. By Thm. 10, we assume that the transition relations are restricted s.t. $R^{\text {may }} \subseteq \operatorname{MT}(P) \times \operatorname{MT}(P)$, and $R^{\text {must }} \subseteq \operatorname{MT}(P) \times \operatorname{Mon}(P)$.

The algorithm uses the following sets of BDD variables: $B=\left\{b_{i} \mid p_{i} \in P\right\}-$ the current state Boolean variables, $B^{\prime}=\left\{b_{i}^{\prime} \mid p_{i} \in P\right\}-$ the next state Boolean variables, $H=$ $\left\{h_{i} \mid p_{i} \in P\right\}-$ the current state unknown variables, and
$H^{\prime}=\left\{h_{i}^{\prime} \mid h_{i} \in H\right\}$ - the next state unknown variables. In what follows, we do not distinguish between the BDDs and the corresponding propositional formulas.

A set of minterms $X \subseteq \mathrm{MT}(P)$ is encoded by a propositional formula over $B$, as usual. For example, let $P=$ $\left\{p_{1}, p_{2}, p_{3}\right\}$. Then $b_{1} \wedge \neg b_{2}$ encodes the set $\left\{p_{1} \wedge \neg p_{2} \wedge p_{3}, p_{1} \wedge\right.$ $\left.\neg p_{2} \wedge \neg p_{3}\right\}$. A set of monomials $X \subseteq \operatorname{Mon}(P)$ is encoded by a formula over $B \cup H$ as follows:

$$
\begin{aligned}
\bigvee_{m \in X}( & \left(\bigwedge_{p_{i} \in \operatorname{Lit}(m)} \neg h_{i} \wedge b_{i}\right) \wedge \\
& \left.\left(\bigwedge_{\neg p_{i} \in \operatorname{Lit}(m)} \neg h_{i} \wedge \neg b_{i}\right) \wedge\left(\bigwedge_{p_{i} \in P \backslash \operatorname{Term}(m)} h_{i}\right)\right)
\end{aligned}
$$

Intuitively, given a monomial $m$, a variable $h_{i}$ indicates whether $p_{i}$ is present in $m$, and a variable $b_{i}$ specifies the polarity of the occurrence. For example, $\left(\neg h_{1} \wedge b_{1}\right) \wedge\left(\neg h_{2} \wedge b_{2}\right) \wedge h_{3}$ represents a singleton set $\left\{p_{1} \wedge \neg p_{2}\right\}$.

An abstract value $e=\langle U, O\rangle$ is encoded in a single BDD by a formula $(\operatorname{sel} \wedge U) \vee(\neg \operatorname{sel} \wedge O)$, where sel is a designated BDD variable. This encoding is implemented by ABSV. $U$ and $O$ elements of the pair are extracted using ABSU and ABSO, respectively. Abstract intersection (ABSAND), union (ABSOR), and equality (ABSEQ) are done using the corresponding BDD operations. Abstract negation (ABSNOT) is implemented following its definition in Sec. II.

The may transition relation $R^{\text {may }} \subseteq \mathrm{MT}(P) \times \operatorname{MT}(P)$ is encoded by a formula over $B \cup B^{\prime}$ as usual. Similarly, the must relation $R^{\text {must }} \subseteq \operatorname{MT}(P) \times \operatorname{Mon}(P)$ is encoded by a formula over $B \cup B^{\prime} \cup H^{\prime}$, where the primed variables are used to encode the destination state. For example, a must transition from a state $\left(p_{1} \wedge p_{2} \wedge p_{3}\right)$ to a state $\left(p_{1} \wedge \neg p_{2}\right)$ is represented by $\left(b_{1} \wedge b_{2} \wedge b_{3}\right) \wedge\left(\left(\neg u_{1}^{\prime} \wedge b_{1}^{\prime}\right) \wedge\left(\neg u_{2}^{\prime} \wedge \neg b_{2}^{\prime}\right) \wedge u_{3}^{\prime}\right)$.

ABSREDU implements the redu reduction operator of Sec. V-C, using the following observation: let $Q \subseteq \operatorname{Mon}(P)$ be a monotone subset, and $a \in \operatorname{Mon}(P)$. If $a \in \operatorname{MT}(P)$, then $\uparrow a \subseteq Q \Leftrightarrow a \in Q$; otherwise, $\uparrow a \subseteq Q$ iff $\uparrow(a \wedge p) \subseteq Q$ and $\uparrow(a \wedge \neg p) \subseteq Q$, where $p$ is a term not occurring in $a$. ABSREDU applies this reasoning recursively on the input diagram. It uses a function UVAR to find a variable $h_{i} \in H$ 
for each variable $b_{i} \in B$. The function ABSPRE implements the pre-image computation based on Thm. 10.

Theorem 11 For a monotone MixTS $\mathcal{M}$ and $\varphi \in L_{\mu}$, the function $\operatorname{RCS}(\varphi)$ returns the symbolic representation of $\|\varphi\|_{r}^{\mathcal{M}}$.

\section{EXPERIMENTS}

We have implemented symbolic algorithms for computing both SCS and RCS using the CUDD [22] library. Our goal was to evaluate the cost and performance of RCS versus SCS on a realistic model. Understanding and analyzing RCS in the context of abstraction refinement and software model-checking is left for future work.

For the model, we used a template program built out of $n$ blocks, each based on an example from [20] and having one integer variable. The method of [11] was applied to build an abstract MixTS via predicate abstraction. We checked one reachability (least fixed-point) property, Prop ${ }_{1}$, and two non-termination (greatest fixed-point) properties, $\mathrm{Prop}_{2}$, and Prop $_{3}$. The code for the experiments is available from http: //www.cs.toronto.edu/ $\sim$ owei/MixTS/FMCAD08.html.

The results are summarized in Fig. 5. The top part of the table shows that, as expected, the model for RCS is significantly smaller, in the \# of DD nodes, than the model for SCS. RCS is always more precise than SCS, and the extra precision changes the number of iterations of the fixpoint computation. For Prop ${ }_{1}$, RCS requires more iterations, and takes more time than SCS. For Prop 2 , RCS is a lot faster than SCS - the fixpoint computation in the former converges in just two steps, whereas the number of iterations in latter is proportional to the size of the model. For $\mathrm{Prop}_{3}$, where RCS and SCS take the same number of iterations, RCS performs significantly better. In all the cases, the time spent in ABSREDU, which represents the main difference between the two semantics, is only about $20 \%-25 \%$ of the total time.

These experiments suggest that the additional precision of RCS improves the overall performance of model checking, making it a viable alternative to SCS in practice.

\section{RELATED WORK AND CONCLUSION}

Related Work. Godefroid and Jagadeesan [9], and Gurfinkel and Chechik [10] proved that the models in the KMTS family have the same expressive power and are equally precise for SCS. Dams and Namjoshi [6] showed that the three families considered in this paper are subsumed by tree automata. Our paper completes the picture by proving that the three families are equivalent as well. Specifically, we showed that KMTSs, MixTSs and GKMTSs are relatively complete (in the sense of [6]) with one another.

In this paper, we did not consider Hyper TSs (HTSs) [21] which allow for both must and may hyper-transitions. As pointed out in [21], may hyper-transitions can be eliminated by increasing the abstract statespace, making HTSs exactly as expressive as GKMTSs.

Our reduction operator RED is an instance of normalization from Abstract Interpretation [4], typically used to provide a

\begin{tabular}{|c|c|c|c|c|c|c|}
\hline & $n$ & \multicolumn{2}{|c|}{ SCS } & \multicolumn{3}{|c|}{ RCS } \\
\hline $\begin{array}{l}\frac{\bar{\pi}}{0} \\
\dot{\Sigma}: \tilde{N}\end{array}$ & \begin{tabular}{|l|}
100 \\
150 \\
200 \\
250 \\
\end{tabular} & $\begin{array}{r}370 \\
825 \\
1,46 \\
2,27\end{array}$ & $\begin{array}{l}070 \\
112 \\
270 \\
196 \\
\end{array}$ & & $\begin{array}{c}216,689 \\
482,531 \\
853,389 \\
1,329,215\end{array}$ & \\
\hline Prop. & $n$ & $\begin{array}{c}\begin{array}{c}\text { Analysis } \\
\text { (sec.) }\end{array} \\
\end{array}$ & $\begin{array}{l}\text { Num. of } \\
\text { Iterations }\end{array}$ & $\begin{array}{c}\text { Analysis } \\
\text { (sec.) }\end{array}$ & $\begin{array}{c}\text { ABSREDU } \\
\text { (sec.) }\end{array}$ & $\begin{array}{l}\text { Num. of } \\
\text { Iterations }\end{array}$ \\
\hline $\begin{array}{l}\overrightarrow{0} \\
\text { Oे } \\
\text { م. }\end{array}$ & \begin{tabular}{|l|}
100 \\
150 \\
200 \\
250 \\
\end{tabular} & $\begin{array}{c}2.20 \\
6.66 \\
15.36 \\
28.92 \\
\end{array}$ & $\begin{array}{l}301 \\
451 \\
601 \\
751 \\
\end{array}$ & $\begin{array}{c}3.60 \\
12.12 \\
27.77 \\
55.19 \\
\end{array}$ & $\begin{array}{c}0.74 \\
2.57 \\
6.45 \\
13.40 \\
\end{array}$ & $\begin{array}{c}401 \\
601 \\
801 \\
1001 \\
\end{array}$ \\
\hline 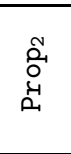 & \begin{tabular}{|l|}
100 \\
150 \\
200 \\
250 \\
\end{tabular} & $\begin{array}{c}3.60 \\
11.91 \\
27.16 \\
54.62 \\
\end{array}$ & $\begin{array}{l}203 \\
303 \\
403 \\
503\end{array}$ & $\begin{array}{l}0.03 \\
0.07 \\
0.12 \\
0.19\end{array}$ & $\begin{array}{l}<10^{-4} \\
<10^{-4} \\
<10^{-4} \\
<10^{-4}\end{array}$ & $\begin{array}{l}2 \\
2 \\
2 \\
2\end{array}$ \\
\hline 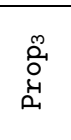 & \begin{tabular}{|l|}
100 \\
150 \\
200 \\
250
\end{tabular} & \begin{tabular}{|c|}
33.96 \\
137.38 \\
395.24 \\
1108.67
\end{tabular} & $\begin{array}{c}400 \\
600 \\
800 \\
1000\end{array}$ & $\begin{array}{c}21.24 \\
76.38 \\
258.72 \\
546.88\end{array}$ & $\begin{array}{c}4.5 \\
15.77 \\
42.44 \\
101.20\end{array}$ & $\begin{array}{c}400 \\
600 \\
800 \\
1000\end{array}$ \\
\hline
\end{tabular}

Fig. 5. Experimental results for SCS and RCS.

canonical representation of equivalent abstract properties. Our symbolic implementation ABSREDU is similar to the semantic minimization of 3-valued propositional formulas [19].

Since RCS is compositional, its precision is between SCS and thorough semantics. Thus, existing results comparing SCS and thorough semantics, i.e., $[8,10,18]$, apply to RCS as well. It is interesting to investigate whether the additional precision enjoyed by RCS can be used to improve the above results.

Summary. In this paper, we compared three families of partial modeling formalisms: KMTSs, MixTSs and GKMTSs. We showed that they are equally expressive - a model of one formalism can be transformed into an equivalent model of another. Thus, neither hyper-transitions nor restrictions on may and must transitions affect expressiveness; they only affect the succinctness of the formalism.

We also proposed a reduced compositional semantics for partial models. This semantics is more precise than the standard one, MixTSs and GKMTSs are equivalent w.r.t. it. We also provided a symbolic implementation of the new semantics, and our experiments suggested that the new semantics is a good alternative to the standard one for predicate abstractionbased model-checkers.

Acknowledgments. We thank Sagar Chaki for comments on earlier drafts of this paper.

\section{REFERENCES}

[1] G. Bruns and P. Godefroid. "Model Checking Partial State Spaces with 3-Valued Temporal Logics". In CAV, vol. 1633 of LNCS, pp. 274-287, 1999.

[2] G. Bruns and P. Godefroid. "Generalized Model Checking: Reasoning about Partial State Spaces". In CONCUR, vol. 1877 of LNCS, pp. 168$182,2000$.

[3] M. Chechik, B. Devereux, S. Easterbrook, and A. Gurfinkel. "MultiValued Symbolic Model-Checking". ACM TOSEM, 12(4):1-38, 2003.

[4] P. Cousot and R. Cousot. "Abstract Interpretation Frameworks". J. of Logic and Computation, 2(4):511-547, 1992.

[5] D. Dams, R. Gerth, and O. Grumberg. "Abstract Interpretation of Reactive Systems". ACM TOPLAS, 2(19):253-291, 1997.

[6] D. Dams and K. S. Namjoshi. "Automata as Abstractions". In VMCAI, vol. 3385 of $L N C S$, pp. 216-232, 2005. 
[7] L. de Alfaro, P. Godefroid, and R. Jagadeesan. "Three-Valued Abstractions of Games: Uncertainty, but with Precision". In LICS, pp. 170-179, 2004.

[8] P. Godefroid and M. Huth. "Model Checking v.s. Generalized Model Checking: Semantic Minimizations for Temporal Logics". In LICS, pp. 158-167, 2005.

[9] P. Godefroid and R. Jagadeesan. "On the Expressiveness of 3-Valued Models". In VMCAI, vol. 2575 of LNCS, pp. 206-222, 2003.

[10] A. Gurfinkel and M. Chechik. "How Thorough is Thorough Enough", In CHARME, vol. 3725 of $L N C S$, pp. 65-80, 2005.

[11] A. Gurfinkel and M. Chechik. "Why Waste a Perfectly Good Abstraction?". In TACAS, vol. 3920 of $L N C S$, pp. 212-226, 2006.

[12] A. Gurfinkel, O. Wei, and M. Chechik. "Systematic Construction of Abstractions for Model-Checking". In VMCAI, vol. 3855 of LNCS, pp. 381-397, 2006.

[13] A. Gurfinkel, O. Wei, and M. Chechik. "YASM: A Software ModelChecker for Verification and Refutation". In CAV, vol. 4144 of LNCS, pp. 170-174, 2006.

[14] M. Huth, R. Jagadeesan, and D. A. Schmidt. "Modal Transition Systems: A Foundation for Three-Valued Program Analysis”. In ESOP, vol. 2028 of $L N C S$, pp. 155-169, 2001.

[15] D. Kozen. "Results on the Propositional $\mu$-calculus". Theoretical Computer Science, 27:334-354, 1983.

[16] K. Larsen and B. Thomsen. "A Modal Process Logic". In LICS, pp. 203-210, 1988.

[17] P. Larsen. "The Expressive Power of Implicit Specifications". In ICALP, vol. 510 of $L N C S$, pp. 204-216, 1991.

[18] S. Nejati, M. Gheorghiu, and M. Chechik. "Thorough Checking Revisited”. In FMCAD, pp. 106-116, 2006.

[19] T. W. Reps, A. Loginov, and S. Sagiv. "Semantic Minimization of 3 Valued Propositional Formulae". In LICS, pp. 40-54, 2002.

[20] S. Shoham and O. Grumberg. "Monotonic Abstraction-Refinement for CTL". In TACAS, vol. 2988 of LNCS, pp. 546-560, 2004.

[21] S. Shoham and O. Grumberg. "3-Valued Abstraction: More Precision at Less Cost". In LICS, pp. 399-410, 2006.

[22] F. Somenzi. "CUDD: CU Decision Diagram Package Release", 2001.

\section{APPENDIX}

The appendix contains proof sketches of theorems for the convenience of the reviewers.

Theorem 2 Let $G$ be a GKMTS, and $M=\operatorname{GTOM}(G)$. Then, $M$ is a MixTS, and $G$ and $M$ are semantically equivalent.

Proof: (1) According to the construction in Def. 6, every must hyper-transition is replaced by a regular one. It is easy to show that $M$ is a MixTS. (2) To prove that $G$ and $M$ are semantically equivalent, we show that any concrete BTS $B=$ $\langle C, R\rangle$ refines $G$ iff it refines $M$. It is equivalent to showing that the soundness relation $\rho_{G} \subseteq C \times S_{G}$ is a mixed simulation between $B$ and $G$ iff the soundness relation $\rho_{M}: C \times S_{M}$ is a mixed simulation between $B$ and $M$. This is proved based on the construction of transition relations given in Def. 6 .

Theorem 3 Let $M=\left\langle S_{M}, R_{M}^{\text {must }}, R_{M}^{\text {may }}\right\rangle$ be a monotone (Def. 9) MixTS transition system, then the following are equivalent:

1) $M$ is semantically consistent, i.e., $\mathbb{C}[M]$ is not empty.

2) $M$ is logically consistent, i.e., $\langle M, L\rangle$ is logically consistent for every (consistent) labeling function $L$.

3) for any $a, b_{1} \in S_{M}, a \stackrel{\text { must }}{\longrightarrow} b_{1} \Rightarrow \exists b_{2} \in S_{M} \cdot b_{1} \preceq$ $b_{2} \wedge a \stackrel{\text { may }}{\longrightarrow} b_{2}$.

Proof:

- (1) $\Rightarrow(2)$. Trivial (by Theorem 2)

- $(2) \Rightarrow(3)$. Given $a \stackrel{\text { must }}{\longrightarrow} b_{1}$, we prove the result by constructing a consistent labeling function $L$ such that for some atomic proposition $p$, to ensure the value $\|\nabla p\|(a)$ is consistent, i.e., belonging to $\{\mathrm{t}, \mathrm{f}, \mathrm{m}\}, a$ must have a may transition to some state $b_{2}$ such that $b_{1} \preceq b_{2}$.

We construct the labeling function $L$ as follows.

We define three sets $S_{1}, S_{2}$, and $S_{3}$.

$$
S_{1}=\left\{s \in S_{M} \mid b_{1} \preceq s\right\}
$$

That is, $S_{1}$ is the set of states which are more precise than $b_{1}$. Let

$$
S_{2}=\left\{s \in S_{M} \mid \exists t \in S_{1} \cdot s \preceq t\right\} \backslash S_{1}
$$

That is, $S_{2}$ is the set of states which are less precise than some state in $S_{1}$, but does not include the states in $S_{1}$ themselves. Let

$$
S_{3}=S_{M} \backslash\left(S_{1} \cup S_{2}\right)
$$

That is, the set of states which are outside of $S_{1}$ and $S_{2}$. Note that $S_{1}, S_{2}$ and $S_{2}$ are disjoint.

Let $A P=\{p\}$ and the labeling function $L$ is defined as follows:

$$
L(s)= \begin{cases}\{p\} & \text { if } s \in S_{1} \\ \{\} & \text { if } s \in S_{2} \\ \{\neg p\} & \text { if } s \in S_{3}\end{cases}
$$

That is, $p$ is true in $S_{1}$, unknown in $S_{2}$, and false in $S_{3}$. We show that $L$ is consistent:

- at most one of $p$ or $\neg p$ belongs to $L(s)$ for any $s \in S_{M}$

- $L$ is monotone, which follows from the following fact: for any $s, t \in S_{M}$

1) $s, t \in S_{1}$ :

In this case $L(s)=L(t)$, and the monotonicity condition is always satisfied. Same result holds for the cases of $s, t \in S_{2}$, and $s, t \in S_{3}$.

2) $s \in S_{1}$ and $t \in S_{2}$ : In this case, suppose $s \preceq t$, then $b_{1} \preceq t$, and $t \in S_{1}$; contradiction.

Therefore, the only possible ordering relation between $s$ and $t$ is $t \preceq s$. Since $L(t) \subseteq L(s)$, the monotonicity condition is satisfied.

3) $s \in S_{2}$ and $t \in S_{3}$ :

In this case, suppose $t \preceq s$, then there exists $s^{\prime} \in S_{1}$, and $t \preceq s^{\prime}$, i.e., $t \in S_{2}$; contradiction.

Therefore, the only possible ordering relation between $s$ and $t$ is $s \preceq t$. Since $L(s) \subseteq L(t)$, monotone condition holds.

4) $s \in S_{3}$ and $t \in S_{1}$ :

In this case, suppose $s \preceq t$, then $s \in S_{2}$; a contradiction. Moreover, suppose $t \preceq s$, then $s \in S_{1}$; a contradiction. Therefore, neither $s \preceq t$ nor $t \preceq s$ holds. Monotone condition is satisfied.

We now show that

$$
\exists b_{2} \in S_{M} \cdot b_{1} \preceq b_{2} \wedge a \stackrel{\text { may }}{\longrightarrow} b_{2}
$$




$$
\begin{array}{ll} 
& a \stackrel{\text { must }}{\longrightarrow} b_{1} \\
\Rightarrow & \text { (by definition of } \left.L,\|p\|=\left\langle S_{1}, S_{1} \cup S_{2}\right\rangle\right) \\
& a \stackrel{\text { must }}{\longrightarrow} b_{1} \wedge b_{1} \in \mathrm{U}(\|p\|) \\
\Rightarrow \quad & (\text { by definition of }\|\triangleright p\|) \\
& a \in \mathrm{U}(\|\triangleright p\|) \\
\Rightarrow \quad & (\text { since }\|\triangleright p\|(a) \text { is consistent) } \\
& a \in \mathrm{O}(\|\triangleright p\|) \\
\Rightarrow \quad & \text { (by definition of }\|\triangleright p\|) \\
& \exists b \in S_{1} \cup S_{2} \cdot a \stackrel{\text { may }}{\longrightarrow} b
\end{array}
$$

Consider different cases of $b$,

- $b \in S_{1}$ : by definition of $S_{1}, b_{1} \preceq b$; let $b_{2}=b$, then (1) holds.

- $b \in S_{2}$ : in this case, we have that

$$
\begin{aligned}
& \exists b \in S_{2} \cdot a \stackrel{\text { may }}{\longrightarrow} b \\
\Rightarrow \quad & \left(\text { by definition of } S_{2}\right. \text { ) } \\
& \exists b \in S_{2} \cdot a \stackrel{\text { may }}{\longrightarrow} b \wedge \exists b^{\prime} \in S_{1} \cdot b \preceq b^{\prime} \\
\Rightarrow \quad & (\text { by monotonicity of } M) \\
& \exists b^{\prime} \in S_{1} \cdot a \stackrel{\text { may }}{\longrightarrow} b^{\prime} \\
\Rightarrow \quad & \text { (by definition of } S_{1} \text { ) } \\
& \exists b^{\prime} \in S_{1} \cdot a \stackrel{\text { may }}{\longrightarrow} b^{\prime} \wedge b_{1} \preceq b^{\prime}
\end{aligned}
$$

let $b_{2}=b^{\prime}$, then (1) holds.

- $(3) \Rightarrow(1)$ : we prove the result by constructing a BTS $B=\langle C, R\rangle$ such that $M \preceq_{\rho} B$, where $C$ is the concrete statespace approximated by $S_{M}$ and $\rho \subseteq C \times S_{M}$ is the soundness relation.

The BTS $B$ be defined as follows: for any $c, d \in C$,

$$
(c, d) \in R \Leftrightarrow \exists b \in S_{M} \cdot \alpha(c) \stackrel{\text { may }}{\longrightarrow} b \wedge(d, b) \in \rho
$$

We now show that $M \preceq_{\rho} B$, i.e., $\rho$ is a mixed simulation between $M$ and $B$.

For every $(c, a) \in \rho$, we have the following results

- for any $b \in A$

$$
\begin{aligned}
& a \stackrel{\text { must }}{\longrightarrow} b \\
\Rightarrow & \text { (since } a \preceq \alpha(c), \text { by monotonicity of } M) \\
& \alpha(c) \stackrel{\text { must }}{\longrightarrow} b \\
\Rightarrow & \text { (by assumption) } \\
& \alpha(c) \stackrel{\text { must }}{\longrightarrow} b \wedge \exists b^{\prime} \cdot b \preceq b^{\prime} \wedge \alpha(c) \stackrel{\text { may }}{\longrightarrow} b^{\prime} \\
\Rightarrow & \left(\text { let } d \text { be a state in } \gamma\left(b^{\prime}\right) ; \text { by the construction of } B\right) \\
& \exists b^{\prime} \cdot b \preceq b^{\prime} \wedge c \rightarrow d \wedge\left(d, b^{\prime}\right) \in \rho \\
\Rightarrow & \left(\text { since } b \preceq b^{\prime}, \gamma\left(b^{\prime}\right) \subseteq \gamma(b)\right) \\
& c \rightarrow d \wedge(d, b) \in \rho
\end{aligned} \Rightarrow
$$

- for any $d \in C$, suppose $c \rightarrow d$, i.e., $c \stackrel{\text { may }}{\longrightarrow} d$, then

$$
\begin{aligned}
& c \stackrel{\text { may }}{\longrightarrow} d \\
\Rightarrow \quad & \text { (by the construction of } B) \\
& \exists b \in S_{M} \cdot \alpha(c) \stackrel{\text { may }}{\longrightarrow} b \wedge(d, b) \in \rho \\
\Rightarrow \quad & (\text { since } a \preceq \alpha(c), \text { by monotonicity of } M) \\
& \exists b \in S_{M} \cdot a \stackrel{\text { may }}{\longrightarrow} b \wedge(d, b) \in \rho
\end{aligned}
$$

Theorem 4 Let $M$ be a MixTS, and $K=\operatorname{MroK}(M)$. Then $K$ is a KMTS, and $M$ and $K$ are semantically equivalent.

Proof: (1) The construction in Def. 7 ensures that every must transition in $K$ is matched by a may transition. Therefore, $K$ is a KMTS. (2) To prove that $M$ and $K$ are semantically equivalent, we show that for any concrete BTS $B=\langle C, R\rangle$, the soundness relation $\rho_{M} \subseteq C \times S_{M}$ is a mixed simulation between $B$ and $M$ iff the soundness relation $\rho_{K}: C \times S_{K}$ is a mixed simulation between $B$ and $K$. The proof follows from the construction of transition relations in Def. 7.

Theorem 5 RCS is more precise than SCS: $\|\varphi\|_{c} \preceq_{a}\|\varphi\|_{r}$.

Proof: The proof is by structural induction on $\varphi$. For the base case, it is obvious that for any atomic proposition $p,\|p\|_{c} \equiv_{a}$ $\|p\|_{r}$. In the following, we show the inductive case for $\nabla \varphi$; the proofs of other cases are trivial.

We show that if $\|\varphi\|_{c} \preceq_{a}\|\varphi\|_{r}$, then $\|\nabla \varphi\|_{c} \preceq_{a}\|\nabla \varphi\|_{r}$, i.e.,

$$
\|\varphi\|_{c} \preceq_{a}\|\varphi\|_{r} \Rightarrow \gamma\left(\mathrm{U}\left(\|\nabla \varphi\|_{c}\right)\right) \subseteq \gamma\left(\mathrm{U}\left(\|\nabla \varphi\|_{r}\right)\right)
$$

and

$$
\|\varphi\|_{c} \preceq_{a}\|\varphi\|_{r} \Rightarrow \gamma\left(\overline{\mathrm{O}\left(\|\nabla \varphi\|_{c}\right)}\right) \subseteq \gamma\left(\overline{\mathrm{O}\left(\|\nabla \varphi\|_{r}\right)}\right)
$$

Proof of (2). For any two sets $Q_{1}, Q_{2}$, we have that

$$
\gamma\left(Q_{1}\right) \subseteq \gamma\left(\operatorname{RED}\left(Q_{2}\right)\right) \Rightarrow Q_{1} \subseteq \operatorname{RED} \mathrm{U}\left(Q_{2}\right)
$$

This follows from the following derivation: suppose $Q_{1} \nsubseteq$ $\operatorname{RED}_{\mathrm{U}}\left(Q_{2}\right)$, then there exists a state $s$ s.t. $s \in Q_{1}$ and $s \notin$ $\operatorname{RED}_{\mathrm{U}}\left(Q_{2}\right)$. By the definition of REDu,$\gamma(s) \nsubseteq \gamma\left(Q_{2}\right)$; on the other hand, since $\gamma\left(Q_{1}\right) \subseteq \gamma\left(\operatorname{RED}_{\mathrm{U}}\left(Q_{2}\right)\right)=\gamma\left(Q_{2}\right), \gamma(s) \subseteq$ $\gamma\left(Q_{2}\right)$. A contradiction.

The proof of (2) is shown below.

$$
\begin{aligned}
& \|\varphi\|_{c} \preceq a\|\varphi\|_{r} \\
& \Rightarrow \quad \text { (by the definition of } \preceq_{a} \text { ) } \\
& \gamma\left(\mathrm{U}\left(\|\varphi\|_{c}\right) \subseteq \gamma\left(\mathrm{U}\left(\|\varphi\|_{r}\right)\right)\right. \\
& \Rightarrow \quad(\text { since } \gamma(Q)=\gamma(\operatorname{RED}(Q))) \\
& \gamma\left(\mathrm{U}\left(\|\varphi\|_{c}\right) \subseteq \gamma\left(\operatorname{RED}_{\mathrm{U}}\left(\mathrm{U}\left(\|\varphi\|_{r}\right)\right)\right)\right. \\
& \Rightarrow \quad \text { (by }(\mathbf{P 1}) \text { ) } \\
& \left.\mathrm{U}\left(\|\varphi\|_{c}\right)\right) \subseteq \operatorname{RED}\left(\mathrm{U}\left(\|\varphi\|_{r}\right)\right) \\
& \Rightarrow \quad\left(\text { since } Q_{1} \subseteq Q_{2} \Rightarrow \operatorname{pre}_{\mathrm{U}}\left(Q_{1}\right) \subseteq \operatorname{pre}_{\mathrm{U}}\left(Q_{2}\right)\right) \\
& \operatorname{pre}_{\mathrm{U}}\left(\mathrm{U}\left(\|\varphi\|_{c}\right)\right) \subseteq \operatorname{pre}_{\mathrm{U}}\left(\operatorname{RED}_{\mathrm{U}}\left(\mathrm{U}\left(\|\varphi\|_{r}\right)\right)\right) \\
& \Rightarrow \quad \text { (by the definition of } \gamma \text { ) } \\
& \gamma\left(\operatorname{preu}_{\mathrm{U}}\left(\mathrm{U}\left(\|\varphi\|_{c}\right)\right)\right) \subseteq \gamma\left(\operatorname{preu}_{\mathrm{U}}\left(\operatorname{REDU}\left(\mathrm{U}\left(\|\varphi\|_{r}\right)\right)\right)\right) \\
& \text { (since } \gamma(Q)=\gamma\left(\operatorname{RED}_{\mathrm{U}}(Q)\right) \\
& \gamma\left(\operatorname{pre}_{\mathrm{U}}\left(\mathrm{U}\left(\|\varphi\|_{c}\right)\right)\right) \subseteq \gamma\left(\operatorname{RED}_{\mathrm{U}}\left(\operatorname{pre}_{\mathrm{U}}\left(\operatorname{RED}_{\mathrm{U}}\left(\mathrm{U}\left(\|\varphi\|_{r}\right)\right)\right)\right)\right) \\
& \Rightarrow \quad \text { (by the definition of SCS and RCS) } \\
& \gamma\left(\mathrm{U}\left(\|\nabla \varphi\|_{c}\right)\right) \subseteq \gamma\left(\mathrm{U}\left(\|\nabla \varphi\|_{r}\right)\right)
\end{aligned}
$$

Proof of (3). Dual of the one above.

Theorem 6 Let $\mathcal{G}$ be a GKMTS, and $\mathcal{M}=\operatorname{GTOM}(\mathcal{G})$. Then, $\mathcal{G}$ and $\mathcal{M}$ are equivalent w.r.t. RCS: $\forall \varphi \in L_{\mu} \cdot\|\varphi\|_{r}^{\mathcal{G}} \equiv_{a}\|\varphi\|_{r}^{\mathcal{M}}$. Proof: The proof is by structural induction on $\varphi$. For the base case, according to the definition of $L_{M}$, we have that for any atomic proposition $p,\|p\|_{r}^{\mathcal{G}} \equiv_{a}\|p\|_{r}^{\mathcal{M}}$. In the following, we show the inductive case for $\nabla \varphi$; the proofs of the other cases are trivial. 

i.e.,

We show that if $\|\varphi\|_{r}^{\mathcal{G}} \equiv_{a}\|\varphi\|_{r}^{\mathcal{M}} \Rightarrow\|\nabla \varphi\|_{r}^{\mathcal{G}} \equiv_{a}\|\diamond\|_{r} \varphi^{\mathcal{M}}$,

$$
\|\varphi\|_{r}^{\mathcal{G}} \equiv_{a}\|\varphi\|_{r}^{\mathcal{M}} \Rightarrow \gamma\left(\mathrm{U}\left(\|\nabla \varphi\|_{r}^{\mathcal{G}}\right)\right) \subseteq \gamma\left(\mathrm{U}\left(\|\nabla \varphi\|_{r}^{\mathcal{M}}\right)\right)
$$

and

$$
\|\varphi\|_{r}^{\mathcal{G}} \equiv{ }_{a}\|\varphi\|_{r}^{\mathcal{M}} \Rightarrow \gamma\left(\overline{\mathrm{O}\left(\|\nabla \varphi\|_{r}^{\mathcal{G}}\right)}\right) \subseteq \gamma\left(\overline{\mathrm{O}\left(\|\nabla \varphi\|_{r}^{\mathcal{M}}\right)}\right)
$$

Proof of (4). For any concrete state $c$ and a set of abstract states $Q$,

$$
c \in \gamma\left(\operatorname{RED}_{\mathrm{U}}(Q)\right) \Leftrightarrow \exists a \in Q \cdot c \in \gamma(a)
$$

The proof of (4) is as follows: for any concrete state $c$,

$$
\begin{aligned}
& c \in \gamma\left(\mathrm{U}\left(\|\nabla \varphi\|_{r}^{\mathcal{G}}\right)\right) \\
& \Leftrightarrow \quad \text { (by the definition of RCS) } \\
& c \in \gamma\left(\operatorname{RED}_{\mathrm{U}}\left(\text { pre }_{\mathrm{U}}^{G}\left(\operatorname{RED}_{\mathrm{U}}\left(\mathrm{U}\left(\|\varphi\|_{r}^{\mathcal{G}}\right)\right)\right)\right)\right) \\
& \Leftrightarrow \quad(\Leftrightarrow) \text { let } a \text { be the abstract state in (P2), } \\
& \left.(\Leftarrow) \text { since } \gamma(Q)=\gamma\left(\operatorname{RED}_{\mathrm{U}}(Q)\right)\right) \\
& c \in \gamma(a) \wedge a \in \operatorname{pre}_{\mathrm{U}}^{G}\left(\operatorname{RED}_{\mathrm{U}}\left(\mathrm{U}\left(\|\varphi\|_{r}^{\mathcal{G}}\right)\right)\right) \\
& \Leftrightarrow \quad \text { (by the definition of preu) } \\
& c \in \gamma(a) \wedge \exists Q \subseteq \operatorname{RED} \mathrm{U}\left(\mathrm{U}\left(\|\varphi\|_{r}^{\mathcal{G}}\right)\right) \cdot R_{G}^{\text {must }}(a, Q) \\
& \Leftrightarrow \quad \text { (by the construction in Def. } 6 \text { and (P2)) } \\
& c \in \gamma(a) \wedge \exists b \cdot \gamma(b) \subseteq \gamma\left(\operatorname{RED}_{\mathrm{U}}\left(\mathrm{U}\left(\|\varphi\|_{r}^{\mathcal{G}}\right)\right)\right) \wedge R_{M}^{\text {must }}(a, b) \\
& \Leftrightarrow \quad\left(\text { since }\|\varphi\|_{r}^{\mathcal{G}} \equiv_{a}\|\varphi\|_{r}^{\mathcal{M}}, \gamma\left(\mathrm{U}\left(\|\varphi\|_{r}^{\mathcal{G}}\right)\right)=\gamma\left(\mathrm{U}\left(\|\varphi\|_{r}^{\mathcal{M}}\right)\right)\right) \\
& c \in \gamma(a) \wedge \exists b \cdot \gamma(b) \subseteq \gamma\left(\operatorname{RED}_{\mathrm{U}}\left(\mathrm{U}\left(\|\varphi\|_{r}^{\mathcal{M}}\right)\right)\right) \wedge R_{M}^{\text {must }}(a, b) \\
& \Leftrightarrow \quad\left(\text { since } \gamma(Q)=\gamma\left(\operatorname{RED}_{\mathrm{U}}(Q)\right) \text {, by the definition of } \mathrm{RED}_{\mathrm{U}}\right) \\
& c \in \gamma(a) \wedge \exists b \in \operatorname{RED} \mathrm{U}\left(\mathrm{U}\left(\|\varphi\|_{r}^{\mathcal{M}}\right)\right) \cdot R_{M}^{\text {must }}(a, b) \\
& \Leftrightarrow \quad \text { (by the definition of } \text { pre }_{\mathrm{U}} \text { ) } \\
& c \in \gamma(a) \wedge a \in \operatorname{pre}_{\mathrm{U}}^{M}\left(\operatorname{RED}_{\mathrm{U}}\left(\mathrm{U}\left(\|\varphi\|_{r}^{\mathcal{M}}\right)\right)\right) \\
& \Leftrightarrow \quad\left((\Rightarrow) \text { since } \gamma(Q)=\gamma\left(\operatorname{REDU}_{\mathrm{U}}(Q)\right)\right. \text {, } \\
& (\Leftarrow) \text { let } a \text { be the abstract state in }(\mathbf{P 2}) \text { ) } \\
& c \in \gamma\left(\operatorname{RED}_{\mathrm{U}}\left(\operatorname{pre}_{\mathrm{U}}^{M}\left(\operatorname{RED}_{\mathrm{U}}\left(\mathrm{U}\left(\|\varphi\|_{r}^{\mathcal{G}}\right)\right)\right)\right)\right) \\
& \Leftrightarrow \quad \text { (by the definition of RCS) } \\
& c \in \gamma\left(\mathrm{U}\left(\|\nabla \varphi\|_{r}^{\mathcal{M}}\right)\right)
\end{aligned}
$$

Proof of (5). The proof is similar to the one above. It is based on the observation that for any concrete state $c$ and a set of abstract states $Q, c \in \gamma\left(\overline{\operatorname{RED}_{\mathrm{O}}(Q)}\right) \Leftrightarrow \exists a \in \bar{Q} \cdot c \in \gamma(a)$.

Theorem 7 Let $S$ be an abstract statespace, and $e$ be a monotone element in $2^{S} \times 2^{S}$. Then, $\operatorname{red}(e)=\operatorname{RED}(e)$.

Proof: According to the definition of RED, the proof of the theorem is equivalent to showing that for any $e=\langle U, O\rangle \in$ $2^{S} \times 2^{S}$ and $s \in S$,

$$
\gamma(s) \subseteq \gamma(U) \Leftrightarrow \uparrow s \subseteq U
$$

and

$$
\gamma(s) \nsubseteq \gamma(\bar{O}) \Leftrightarrow \uparrow s \nsubseteq \bar{O}
$$

Proof of $(6)$. The $(\Rightarrow)$ direction follows from the following derivation: supposing that $\uparrow s \nsubseteq U$, we have that

$$
\begin{array}{ll} 
& \uparrow s \nsubseteq U \\
\Rightarrow \quad & \exists a \in S \cdot a \in \uparrow s \wedge a \notin U \\
\Rightarrow \quad & \text { (since } a \in \uparrow s, a \in \alpha[S]) \\
& \exists a \in S \cdot a \in \uparrow s \wedge a \notin U \wedge \exists c \in C \cdot a=\alpha(c) \\
\Rightarrow \quad & \text { (by the def. of } \uparrow, \gamma(a) \subseteq \gamma(s) ; \text { since } \gamma(s) \subseteq \gamma(U)) \\
& \exists a \in S \cdot a \in \uparrow s \wedge a \notin U \wedge \exists c \in C \cdot a=\alpha(c) \wedge \\
& c \in \gamma(U) \\
\Rightarrow \quad & \text { (by the definition of }) \\
& \exists a \in S \cdot a \in \uparrow s \wedge a \notin U \wedge \exists c \in C \cdot a=\alpha(c) \wedge \\
& c \in \gamma(U) \wedge \exists b \in U \cdot c \in \gamma(b) \\
\Rightarrow \quad & \text { (by the definition of } \alpha) \\
& \exists a \in S \cdot a \in \uparrow s \wedge a \notin U \wedge \exists c \in C \cdot a=\alpha(c) \wedge \\
& c \in \gamma(U) \wedge \exists b \in U \cdot c \in \gamma(b) \wedge b \preceq a a \\
\Rightarrow \quad & \text { (by monotonicity of } e, a \in U) \\
& \exists a \in S \cdot a \in \uparrow s \wedge a \notin U \wedge a \in U
\end{array}
$$

A contradiction. The $(\Leftarrow)$ direction is trivial.

Proof of (7). Dual of the one above.

Theorem 8 Let $e_{1}=\left\langle U_{1}, O_{1}\right\rangle$ be a monotone element of $2^{S} \times$ $2^{S}$, and $e_{2}=\left\langle U_{2}, O_{2}\right\rangle$ be in $2^{\alpha[S]} \times 2^{\alpha[S]}$. If $U_{1} \cap \alpha[S]=U_{2}$, and $O_{1} \cap \alpha[S]=O_{2}$, then $e_{1} \equiv{ }_{a} e_{2}$.

Proof: This is proved by showing that $\operatorname{RED}\left(e_{1}\right)=\operatorname{RED}\left(e_{2}\right)$; since RED is semantics preserving, the result holds.

Theorem 9 Let $R^{\text {may }} \subseteq S \times S$ and $R^{\text {must }} \subseteq S \times S$ be the may and must transition relations of a monotone MixTS, respectively, and $e=\langle U, O\rangle$ be a monotone element of $2^{S} \times 2^{S}$. Define $\hat{U} \triangleq U \cap \alpha[S], \hat{O} \triangleq O \cap \alpha[S], \hat{R}^{\text {must }} \triangleq$ $R^{\text {must }} \cap(\alpha[S] \times S)$, and $\hat{R}^{\text {may }} \triangleq R^{\text {may }} \cap(\alpha[S] \times \alpha[S])$. Then,

$$
\begin{array}{r}
\left\langle\operatorname{pre}\left[R^{\text {must }}\right](\operatorname{RED} \mathrm{U}(U)), \operatorname{pre}\left[R^{\text {may }}\right]\left(\operatorname{RED}_{\mathrm{O}}(O)\right)\right\rangle \equiv_{a} \\
\left\langle\text { pre }\left[\hat{R}^{\text {must }}\right]\left(\operatorname{RED}_{\mathrm{U}}(\hat{U})\right), \operatorname{pre}\left[\hat{R}^{\mathrm{may}}\right](\hat{O})\right\rangle
\end{array}
$$

Proof: According to the definition of $\equiv_{a}$, we show that

$$
\begin{gathered}
\gamma\left(\operatorname{pre}\left[R^{\text {must }}\right](\operatorname{RED}(U))\right)=\gamma\left(\operatorname{pre}\left[\hat{R}_{1}\right]\left(\operatorname{RED}_{\mathbf{U}}(\hat{U})\right)\right) \\
\text { and } \quad \gamma\left(\text { pre }\left[R^{\text {may }}\right]\left(\operatorname{RED}_{\mathrm{O}}(O)\right)\right)=\gamma\left(\operatorname{pre}\left[\hat{R}_{2}\right](\hat{O})\right)
\end{gathered}
$$

Proof of (8). The fact that $\gamma\left(\right.$ pre $\left.\left[R^{\text {must }}\right]\left(\operatorname{RED}_{\mathrm{U}}(U)\right)\right) \subseteq$ $\gamma\left(\operatorname{pre}\left[\hat{R}_{1}\right](\operatorname{RED} \mathrm{U}(\hat{U}))\right)$ is shown as follows. For any concrete state $c$,

$c \in \gamma\left(\right.$ pre $\left.\left[R^{\text {must }}\right]\left(\operatorname{RED}_{\mathrm{U}}(U)\right)\right)$

$\Rightarrow \exists a \in S \cdot c \in \gamma(a) \wedge a \in \operatorname{pre}\left[R^{\text {must }}\right]\left(\operatorname{RED}_{\mathrm{U}}(U)\right)$

$\Rightarrow \quad$ (by the definition of pre)

$\exists a \in S \cdot c \in \gamma(a) \wedge \exists b \in \operatorname{RED}_{\mathrm{U}}(U) \cdot R^{\text {must }}(a, b)$

$\Rightarrow \quad$ (let $a^{\prime}=\alpha(c)$, by the definition of $\alpha$ ) $c \in \gamma\left(a^{\prime}\right) \wedge a^{\prime} \in \alpha[S] \wedge \exists a \in S \cdot a \preceq_{a} a^{\prime} \wedge$

$\exists b \in \operatorname{RED}_{\mathrm{U}}(U) \cdot R^{\text {must }}(a, b)$

$\Rightarrow \quad$ (by the definition of monotone MixTSs) $c \in \gamma\left(a^{\prime}\right) \wedge a^{\prime} \in \alpha[S] \wedge \exists b \in \operatorname{RED}_{\mathrm{U}}(U) \cdot R^{\text {must }}\left(a^{\prime}, b\right)$

$\Rightarrow \quad$ (by the definition of $\hat{R}^{\text {must }}$ ) $c \in \gamma\left(a^{\prime}\right) \wedge a^{\prime} \in \alpha[S] \wedge \exists b \in \operatorname{RED}(U) \cdot \hat{R}^{\text {must }}\left(a^{\prime}, b\right)$

$\Rightarrow \quad$ (since $e$ is a monotone element, by Theorem 8) $c \in \gamma\left(a^{\prime}\right) \wedge a^{\prime} \in \alpha[S] \wedge \exists b \in \operatorname{red}_{\mathrm{U}}(U) \cdot \hat{R}^{\text {must }}\left(a^{\prime}, b\right)$

$\Rightarrow \quad$ (by the definition of $\hat{U}, \operatorname{red}_{\mathrm{U}}(U)=\operatorname{red}_{\mathrm{U}}(\hat{U})$ ) $c \in \gamma\left(a^{\prime}\right) \wedge a^{\prime} \in \alpha[S] \wedge \exists b \in \operatorname{red}(\hat{U}) \cdot \hat{R}^{\text {must }}\left(a^{\prime}, b\right)$

$\Rightarrow \quad$ (by the definition of pre) $c \in \gamma\left(a^{\prime}\right) \wedge a^{\prime} \in \operatorname{pre}\left[\hat{R}_{1}\right]\left(\operatorname{RED}_{\mathrm{U}}(\hat{U})\right)$

$\Rightarrow \quad c \in \gamma\left(\right.$ pre $\left.\left[\hat{R}^{\text {must }}\right]\left(\operatorname{RED}_{\mathrm{U}}(U)\right)\right)$ 
The proof of $\gamma\left(\right.$ pre $\left.\left[R^{\text {must }}\right]\left(\operatorname{RED}_{\mathrm{U}}(U)\right)\right) \supseteq$ $\gamma\left(\right.$ pre $\left.\left[\hat{R}_{1}\right](\operatorname{RED} \mathrm{U}(\hat{U}))\right)$ is trivial.

Proof of (9). The fact that $\gamma\left(\overline{\text { pre }\left[R^{\text {may }}\right]\left(\operatorname{RED}_{\mathrm{O}}(O)\right)}\right) \subseteq$

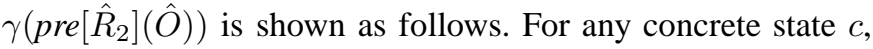
$c \in \gamma\left(\overline{\text { pre }\left[R^{\text {may }}\right]\left(\operatorname{RED}_{\mathrm{O}}(O)\right)}\right)$

$\Rightarrow \exists a \in S \cdot c \in \gamma(a) \wedge a \in \overline{\text { pre }\left[R^{\text {may }}\right]\left(\operatorname{RED}_{\mathrm{O}}(O)\right)}$

$\Rightarrow \quad$ (by the definition of pre) $\exists a \in S \cdot c \in \gamma(a) \wedge R^{\text {may }}(a) \subseteq \overline{\operatorname{RED}_{\mathrm{O}}(O)} \wedge c \in \gamma(a)$

$\Rightarrow \quad\left(\right.$ let $a^{\prime}=\alpha(c)$, by the definition of $\alpha$ ) $c \in \gamma\left(a^{\prime}\right) \wedge a^{\prime} \in \alpha[S] \wedge$

$\exists a \in S \cdot a \preceq_{a} a^{\prime} \wedge R^{\text {may }}(a) \subseteq \overline{\operatorname{RED}_{\mathrm{O}}(O)}$

$\Rightarrow \quad$ (by the definition of monotone MixTSs) $c \in \gamma\left(a^{\prime}\right) \wedge a^{\prime} \in \alpha[S] \wedge$

$\exists a \in S \cdot R^{\text {may }}\left(a^{\prime}\right) \subseteq R^{\text {may }}(a) \subseteq \overline{\operatorname{RED}_{\mathrm{O}}(O)}$

$\Rightarrow \quad$ (by the definition of $\hat{R}^{\text {may }}, R^{\text {may }}\left(a^{\prime}\right) \cap \alpha[S]=\hat{R}^{\text {may }}\left(a^{\prime}\right)$ ) $c \in \gamma\left(a^{\prime}\right) \wedge a^{\prime} \in \alpha[S] \wedge \hat{R}^{\text {may }}\left(a^{\prime}\right) \subseteq\left(\overline{\operatorname{RED}_{\mathrm{O}}(O)} \cap \alpha[S]\right)$

$\Rightarrow \quad$ (since $e$ is a monotone element, by Theorem 8) $\left.c \in \gamma\left(a^{\prime}\right) \wedge a^{\prime} \in \alpha[S] \wedge \hat{R}^{\text {may }}\left(a^{\prime}\right) \subseteq \overline{(\operatorname{redo}(O)} \cap \alpha[S]\right)$

$\Rightarrow \quad$ (by the definition of redo) $\left.\forall s \in \alpha[S] \cdot s \in \operatorname{red}_{\mathrm{O}}(O) \Leftrightarrow s \in O\right)$ $c \in \gamma\left(a^{\prime}\right) \wedge a^{\prime} \in \alpha[S] \wedge \hat{R}^{\text {may }}\left(a^{\prime}\right) \subseteq(\bar{O} \cap \alpha[S])$

$\Rightarrow \quad$ (by the definition of $\hat{O}$ ) $c \in \gamma\left(a^{\prime}\right) \wedge a^{\prime} \in \alpha[S] \wedge \hat{R}^{\text {may }}\left(a^{\prime}\right) \subseteq \alpha[S] \backslash \hat{O}$

$\Rightarrow \quad$ (by the definition of pre) $c \in \gamma\left(\underline{\left.a^{\prime}\right) \wedge a^{\prime} \in \gamma} \overline{\left(\operatorname{pre}\left[\hat{R}_{2}\right](\hat{O})\right.}\right)$

$\Rightarrow \quad c \in \gamma\left(\overline{\operatorname{pre}\left[\hat{R}_{2}\right](\hat{O})}\right)$

The proof of $\gamma\left(\overline{\text { pre }\left[R^{\text {may }}\right]\left(\operatorname{RED}_{\mathrm{O}}(O)\right)}\right) \supseteq \gamma\left(\overline{\operatorname{pre}\left[\hat{R}_{2}\right](\hat{O})}\right)$ is similar.

Theorem 10 For a monotone MixTS $\mathcal{M}$ and $\varphi \in L_{\mu}$, the function $\operatorname{RCS}(\varphi)$ returns the symbolic representation of $\|\varphi\|_{r}^{\mathcal{M}}$.

Proof: The proof follows from Thm. 8, Thm. 9, and Thm. 10. In particular, Thm. 9 is used to show that in the interpretation of $\nabla \varphi$ in Def. 8, removing the application of RED after pre $\mathrm{U}$ and pre $_{\mathrm{O}}$ does not affect precision. 